The AstrophysicAl Journal SupPLEMENT SerIES, 111:181-201, 1997 July

(C) 1997. The American Astronomical Society. All rights reserved. Printed in U.S.A.

\title{
OPTICAL SPECTROSCOPIC PROPERTIES OF A SAMPLE OF INTERACTING GALAXIES
}

\author{
C. J. DONZELLi ${ }^{1}$ \\ IATE, Observatorio Astronómico, Universidad Nacional de Córdoba, Laprida 854, 5000 Córdoba, Argentina; \\ charly@oac.uncor.edu \\ AND \\ M. G. PASTORIZA \\ Departament de Astronomia, Instituto de Física, Universidade Federal do Rio Grande do Sul, CP 15051, \\ Porto Alegre, 91500, RS, Brasil; mgp@if1.ufrgs.br \\ Received 1996 August 26; accepted 1997 January 16
}

\begin{abstract}
We present spectroscopic observations of 83 galaxies from a sample of 49 pairs of optically selected interacting galaxies, most of them previously unobserved. These pairs consist of a main galaxy (component A) and a companion (component B) that has about half or less the diameter of component A.

From our spectra we determine that 27 galaxies form truly physical pairs and seven are apparent pairs, for the remaining pairs we could only extract the spectra of the A components. The spectra of the physical pairs were classified into four groups according to the emission-line spectra observed in each component. These classifications were made because the sample exhibits a very large range of spectral properties, ranging from well-evolved stellar populations (older than $200 \mathrm{Myr}$ ) to emission-linedominated starburst systems ( $80 \mathrm{Myr}$ or younger). In general terms, these spectral types are well correlated with the morphological types of the galaxies. However, we find no evidence of correlation of the equivalent width of $\mathrm{H} \alpha+[\mathrm{N} \mathrm{II}]$ emission lines with the degree of the interaction or with the blue absolute magnitude of the components.

From the data it is also determined that the average $\mathrm{EW}(\mathrm{H} \alpha+[\mathrm{N} \mathrm{II}])$ for the physical pairs is $37 \AA$ for the A components and $54 \AA$ for the B components. For the galaxies that form apparent pairs we obtain $\mathrm{EW}(\mathrm{H} \alpha+[\mathrm{N} \mathrm{II}])=27 \AA$, confirming that physical pairs have higher mean star formation rates than isolated galaxies. This enhancement of the star formation activity is more likely to take place in both galaxies, but the strength of the activity seems to be higher in the B components. The mean observed values of $\mathrm{EW}(\mathrm{H} \alpha+[\mathrm{N} \mathrm{II}])$ are comparable with those observed in a sample of strongly interacting or merging galaxies. On the other hand, we do not find the excess of Seyfert-type nuclei previously reported in studies of similar samples of galaxies.
\end{abstract}

Subject headings: galaxies: interactions — galaxies: photometry — galaxies: Seyfert galaxies: stellar content

\section{INTRODUCTION}

It is widely accepted that interactions can be a significant, if not a dominant, process in galaxy evolution (Karachentsev \& Karachentseva 1974; Wasilewski 1983; Soifer et al. 1984). In fact, after the seminal paper by Toomre \& Toomre (1972) it was realized that the study of interacting galaxies would greatly aid understanding of galaxy formation as well as mechanisms of activity generation and star formation in the nuclear region and/or in the whole galaxy.

Previous works has shown that interacting galaxies are more active in the UV (Larson \& Tinsley 1978), in the near-IR (Joseph \& Wright 1985), in optical emission-line strength (Kennicutt \& Keel 1984), and in radio emission (Stocke 1978; Hummel 1981). In previous papers we have made a comparison of the star formation history in the nucleus of spiral galaxies between a sample in a high galaxy density medium (HDS) and a control sample of isolated galaxies (CS) (Pastoriza et al. 1994; Maia et al. 1994). It was found that the environmental influences in the samples do

${ }^{1}$ Visiting Astronomer at Complejo Astronómico El Leoncito (CASLEO) Observatory. CASLEO is operated under agreement between the Consejo Nacional de Investigaciones Científicas y Técnicas de la República Argentina and the National Universities of La Plata, Córdoba and San Juan not substantially affect the nuclear stellar population. However, the emission lines from the nucleus indicate that there is an excess of HDS galaxies located within or close to, the active galactic nucleus (AGN) loci in the diagnostic diagrams.

On the other hand, it is well known that the star formation rate (SFR) could be enhanced through tidal interactions as a result of close encounters (Bushouse 1986; Kennicutt et al. 1987). Thus, studies of binary or multiple systems of galaxies chosen according to certain parameters (for instance, morphological and spectral types, signs of interactions, and so forth) give excellent opportunities to test scenarios for the origin and evolution of galaxies (e.g., Armus, Heckman, \& Miley 1987; Friedmann et al. 1987; Fried \& Lutz 1988; Bothum et al. 1989; Shombert, Walling, \& Struck-Marcell 1990).

The aims of this work are to present optical spectra and to provide a preliminary analysis of these data for a sample of interacting galaxies, most of which have not previously been observed.

This paper is structured as follows: in $\S 2$ we present the galaxy sample. In $\S 3$ we describe the observations and data reduction. A classification of the sample into physical pairs, apparent pairs, and those with incomplete information, as well as the individual spectra for the galaxy sample, are presented in $\S 4$. In $\S 5$ we present a spectroscopic classification of the physical pairs according to the excitations of 
TABLE 1

ObServed Galaxy Pairs

\begin{tabular}{|c|c|c|c|c|c|c|c|c|}
\hline Name & $\begin{array}{c}\text { AMC } \\
\text { Category }\end{array}$ & R.A. (J1950) & Decl. (J1950) & Type & $m_{b}$ & $\begin{array}{c}c z \\
\left(\mathrm{~km} \mathrm{~s}^{-1}\right)\end{array}$ & $A_{b}$ & Comments \\
\hline AM $0054-411 \ldots \ldots$ & 8 & 005455 & -411355 & S? & 13.99 & 3542 & 0.04 & \\
\hline AM $0103-302 \ldots \ldots$ & 8 & 010349 & -302648 & $\mathrm{SB}(\mathrm{r}) \mathrm{c}$ & 13.80 & 9620 & 0.03 & \\
\hline AM $0134-373 \ldots \ldots$ & $1 d$ & 013411 & -373500 & $\left(\mathrm{R}^{\prime}\right) \mathrm{SA}:(\mathrm{rs}) \mathrm{b}$ & 13.48 & 5148 & 0.03 & \\
\hline AM $0223-403 \ldots \ldots$ & $1 \mathrm{~b}$ & 022313 & -403918 & C pec & & 11812 & 0.07 & \\
\hline AM $0304-391 \ldots \ldots$ & $1 d$ & 030411 & -391342 & SA:(s:)a pec & 13.32 & 6236 & 0.00 & \\
\hline AM $0329-502 \ldots \ldots$ & $1 \mathrm{c}$ & 032913 & -502836 & $\mathrm{SC}$ pec & $\ldots$ & $\ldots$ & 0.00 & \\
\hline AM $0340-280 \ldots \ldots$ & $1 \mathrm{c}$ & 044003 & -280136 & SA pec & $\ldots$ & $\ldots$ & 0.00 & \\
\hline AM $0407-612 \ldots \ldots$ & $1 \mathrm{~b}$ & 040734 & -612412 & $\mathrm{Sb}$ pec & $\ldots$ & $\ldots$ & 0.00 & \\
\hline AM $0409-460 \ldots \ldots$ & 8 & 040951 & -460806 & SA & & 4951 & 0.04 & \\
\hline AM $0547-244 \ldots \ldots$ & $1 \mathrm{c}$ & 054724 & -244706 & s... & 15.59 & $\ldots$ & 0.00 & \\
\hline AM $0550-342 \ldots \ldots$ & 9 & 055031 & -342124 & SB(r)c:pec & 14.11 & 3183 & 0.00 & \\
\hline AM $0728-664 \ldots \ldots$ & $1 \mathrm{~b}$ & 072850 & -664736 & $\mathrm{SAB}(\mathrm{rs}) \mathrm{bc}$ & 13.67 & 5197 & 0.73 & \\
\hline AM $0821-783 \ldots \ldots$ & 8 & 082112 & -783206 & $\mathrm{SA}(\mathrm{s}) \mathrm{b}$ & $\ldots$ & 5545 & 0.37 & \\
\hline AM $0905-232 \ldots \ldots$ & $1 \mathrm{~b}$ & 090527 & -232506 & $\mathrm{Sb}: \mathrm{pec} \mathrm{sp}$ & 14.16 & 3446 & 1.06 & \\
\hline AM $0907-753 \ldots \ldots$ & $1 \mathrm{~b}$ & 090652 & -753630 & $\mathrm{SA}$ & $\ldots$ & 4731 & 0.57 & \\
\hline AM $1023-292 \ldots \ldots$ & $1 d$ & 102328 & -291732 & E pec & & $\ldots$ & 0.28 & Northwest pair \\
\hline AM $1023-292 \ldots \ldots$ & $1 d$ & 102331 & -292054 & Sa: & 14.73 & $\ldots$ & 0.28 & Southeast pair \\
\hline AM $1108-300 \ldots \ldots$ & 9 & 110815 & -300430 & $\mathrm{~S} ?$ & 14.33 & 8881 & 0.35 & \\
\hline AM $1116-290 \ldots \ldots$ & $1 \mathrm{c}$ & 111627 & -290848 & $\mathrm{~S} ?$ & 14.75 & 9064 & 0.39 & Southeast pair \\
\hline AM $1118-350 \ldots \ldots$ & $1 \mathrm{c}$ & 111856 & -350706 & $\mathrm{E} / \mathrm{SO}$ & 15.50 & 9724 & 0.40 & West galaxy \\
\hline AM $1118-350 \ldots \ldots$ & $1 \mathrm{c}$ & 111859 & -350708 & Ec & & $\ldots$ & 0.40 & East pair \\
\hline AM $1127-351 \ldots \ldots$ & 8 & 112736 & -351542 & $\mathrm{Sc}$ & 14.50 & $\ldots$ & 0.31 & \\
\hline AM $1208-273 \ldots \ldots$ & $1 \mathrm{c}$ & 120808 & -273212 & $\mathrm{SBc}$ & $\ldots$ & 14778 & 0.27 & \\
\hline AM $1208-305 \ldots \ldots$ & $1 \mathrm{c}$ & 120833 & -305042 & $\mathrm{~S} ?$ & 13.73 & 2200 & 0.33 & \\
\hline AM $1209-414 \ldots \ldots$ & $1 d$ & 120952 & -414542 & E & $\ldots$ & $\ldots$ & 0.37 & \\
\hline AM $1219-430 \ldots \ldots$ & $1 \mathrm{c}$ & 121918 & -430324 & S pec & & $\ldots$ & 0.57 & \\
\hline AM $1224-331 \ldots \ldots$ & $1 \mathrm{~b}$ & 122445 & -331448 & SA(r)a? & 14.73 & $\ldots$ & 0.51 & \\
\hline AM $1252-443 \ldots \ldots$ & $1 \mathrm{c}$ & 125232 & -443242 & SB?a? & 14.10 & 7061 & 0.43 & \\
\hline AM $1254-321 \ldots \ldots$ & 8 & 125426 & -321442 & $\mathrm{SO} / \mathrm{a}$ & 15.50 & 4730 & 0.36 & \\
\hline AM $1256-433 \ldots \ldots$ & 1a & 125600 & -433625 & E & 14.75 & 8986 & 0.41 & Southwest pair \\
\hline AM $1256-433 \ldots \ldots$ & $1 \mathrm{a}$ & 125607 & -433402 & SBC & 16.02 & 9190 & 0.55 & Northeast pair \\
\hline AM $1304-333 \ldots \ldots$ & 9 & 130421 & -333542 & $\mathrm{Sb}$ pec & & 9260 & 0.28 & \\
\hline AM $1312-365 \ldots \ldots$ & 9 & 131216 & -365254 & $\mathrm{SAB}(\mathrm{r}) \mathrm{ab}$ & 14.23 & 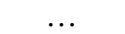 & 0.21 & \\
\hline AM $1344-323 \ldots \ldots$ & $1 d$ & 134436 & -323654 & $E+5$ & 13.01 & 11679 & 0.22 & \\
\hline AM $1401-324 \ldots \ldots$ & $1 d$ & 140119 & -324712 & $\mathrm{Sb}$ & 14.80 & $\ldots$ & 0.25 & \\
\hline AM $1448-262 \ldots \ldots$ & $1 \mathrm{c}$ & 144818 & -262530 & $(\mathrm{R}$ ?)SB(rs)0/a & 14.14 & 2611 & 0.68 & \\
\hline AM $1921-534 \ldots \ldots$ & 8 & 192127 & -534248 & $\left(\mathrm{R}^{\prime}-2\right) \mathrm{SB}(\mathrm{r}) \mathrm{A}$ & 15.43 & 17550 & 0.37 & \\
\hline AM $2011-740 \ldots \ldots$ & 8 & 201137 & -740236 & SB(s)cd & 13.40 & 3159 & 0.28 & \\
\hline AM $2030-303 \ldots \ldots$ & $1 \mathrm{~b}$ & 203053 & -303236 & SA? & & $\ldots$ & 0.33 & West and east galaxies \\
\hline AM $2058-381 \ldots \ldots$ & 9 & 205828 & -381636 & $\mathrm{~S} ?$ & 15.50 & 12154 & 0.13 & \\
\hline AM $2100-381 \ldots \ldots$ & 9 & 210045 & -381636 & $\mathrm{~S} ?$ & $\ldots$ & $\ldots$ & 0.04 & \\
\hline AM $2105-332 \ldots \ldots$ & 9 & 210503 & -332636 & E pec & $\ldots$ & $\ldots$ & 0.08 & \\
\hline AM $2229-735 \ldots \ldots$ & $1 \mathrm{a}$ & 222949 & -735636 & SO? & $\ldots$ & $\ldots$ & 0.07 & \\
\hline AM $2238-575 \ldots \ldots$ & 8 & 223851 & -575731 & $\mathrm{SC}$ & $\ldots$ & $\ldots$ & 0.00 & \\
\hline AM $2302-502 \ldots \ldots$ & 8 & 230222 & -502248 & $\left(\mathrm{R}^{\prime}\right) \mathrm{SAB}(\mathrm{s}) \mathrm{cd}$ & $\ldots$ & 3686 & 0.00 & \\
\hline AM $2304-490 \ldots \ldots$ & $1 \mathrm{c}$ & 230453 & -490112 & Sc: pec & & 8496 & 0.00 & \\
\hline AM $2306-721 \ldots \ldots$ & $1 \mathrm{c}$ & 230620 & -721700 & SC pec & 15.90 & $\ldots$ & 0.09 & \\
\hline AM $2322-821 \ldots \ldots$ & $1 \mathrm{c}$ & 232242 & -821118 & $\mathrm{SA}(\mathrm{r}) \mathrm{bc}$ & 13.23 & 3679 & 0.65 & \\
\hline AM $2330-451 \ldots \ldots$ & $1 \mathrm{~b}$ & 233033 & -451736 & $\mathrm{E}$ & 12.34 & 3121 & 0.05 & \\
\hline AM $2359-275 \ldots \ldots$ & 8 & 235921 & -275412 & $\left(\mathrm{R}^{\prime}\right) \mathrm{SA}(\mathrm{s}) \mathrm{ab}$ & 14.66 & 8507 & 0.05 & \\
\hline
\end{tabular}

Notes.-All data listed refer to the A component. Units of right ascension are hours, minutes, and seconds, and units of declination are degrees, arcminutes, and arcseconds.

the spectra observed in both components. In $\S 6$ we analyze the luminosity distribution, the star formation rates, and the star formation history for the sample galaxies. Finally, some concluding remarks are given in $\S 7$.

\section{SAMPLE SELECTION}

For our study we selected galaxies with a small companion (about half the size of the main galaxy or smaller) from the catalog of Arp \& Madore (1987), hereafter AMC. These pairs exhibit either morphological perturbation of the main galaxy or distortion of the small companion. We specifically chose galaxies from category 1 (galaxies with interacting companions) and category 9 (M51 types), since we are initially interested in studying galaxies with a compact companion; spectroscopic data already exists for pairs of galaxies of comparable sizes (see Sekiguchi \& Wolstencroft 1992, hereafter S\&W). In addition, we would like to shed light on the following questions: how do the properties of these two different samples compare? What are the effects of the interaction between two galaxies with very different masses? Are the age and strength of the bursts of star formation comparable for both galaxies of the pair?

On the other hand, we have also selected galaxies from category 8 (galaxies with apparent companions) in order to compare their properties with those of the real pairs. In this 
TABLE 2

\begin{tabular}{|c|c|c|c|c|}
\hline \multicolumn{5}{|c|}{ Observed Galaxy PaIRs } \\
\hline Name & $\begin{array}{l}\text { Observation } \\
\text { Date }\end{array}$ & $\begin{array}{l}\text { Number of } \\
\text { Frames }\end{array}$ & $\begin{array}{l}\text { Exposure } \\
\text { Time } \\
\text { (s) }\end{array}$ & $\begin{array}{l}\text { Description of } \\
\text { B Component }\end{array}$ \\
\hline 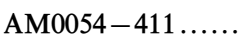 & 1995 Aug 24 & 3 & 600 & \\
\hline AM0103-302..... & 1995 Aug 25 & 5 & 600 & \\
\hline AM0134-373 ..... & 1995 Aug 25 & 5 & 600 & \\
\hline AM0223-403..... & 1994 Oct 01 & 4 & 600 & \\
\hline AM0304-391 ..... & 1995 Aug 25 & 4 & 600 & \\
\hline AM0329-502..... & 1994 Oct 01 & 4 & 600 & \\
\hline AM0340-280..... & 1994 Oct 02 & 5 & 600 & \\
\hline AM0407-612..... & 1994 Oct 02 & 4 & 600 & \\
\hline AM0409-460..... & 1994 Oct 01 & 5 & 600 & \\
\hline 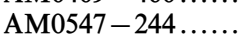 & 1995 Mar 05 & 5 & 600 & \\
\hline AM0550-342..... & 1995 Mar 06 & 5 & 600 & \\
\hline AM0728 $-664 \ldots \ldots$ & 1994 Apr 17 & 6 & 400 & Southeast companion \\
\hline AM0821-783..... & 1994 Apr 15 & 6 & 400 & \\
\hline AM0905-232..... & 1994 Apr 16 & 6 & 400 & West companion \\
\hline AM0907-753..... & 1994 Apr 15 & 6 & 400 & \\
\hline AM1023-292 ..... & 1995 Mar 06 & 5 & 600 & \\
\hline AM1023-292 ..... & 1995 Mar 04 & 5 & 600 & \\
\hline AM1108-300 ..... & 1994 Apr 15 & 6 & 400 & \\
\hline AM1116-290..... & 1994 Apr 16 & 6 & 400 & \\
\hline AM1118-350..... & 1994 Apr 17 & 6 & 400 & \\
\hline AM1118-350..... & 1994 Apr 17 & 5 & 400 & \\
\hline AM1127-351..... & 1994 Apr 17 & 6 & 400 & \\
\hline AM1208-273 ..... & 1994 Apr 16 & 6 & 400 & \\
\hline AM1208-305..... & 1995 Mar 05 & 5 & 600 & \\
\hline AM1209-414 ..... & 1995 Mar 06 & 5 & 600 & \\
\hline AM1219-430..... & 1995 Mar 04 & 4 & 600 & \\
\hline AM1224-331 ..... & 1995 Mar 05 & 5 & 600 & \\
\hline AM1252-443..... & 1994 Apr 15 & 6 & 400 & \\
\hline AM1254-321..... & 1994 Apr 15 & 6 & 400 & Southeast companion \\
\hline AM1256-433 ..... & 1995 Mar 04 & 3 & 600 & \\
\hline AM1256-433..... & 1995 Mar 05 & 3 & 600 & \\
\hline AM1304-333 ..... & 1994 Apr 17 & 6 & 400 & \\
\hline AM1312-365..... & 1995 Mar 06 & 5 & 600 & Northwest companion \\
\hline AM1344-323..... & 1995 Mar 05 & 5 & 600 & \\
\hline AM1401-324..... & 1994 Apr 17 & 7 & 400 & South companion \\
\hline AM1448 $-262 \ldots \ldots$ & 1994 Apr 15 & 6 & 400 & \\
\hline AM1921 $-534 \ldots \ldots$ & 1995 Aug 25 & 3 & 600 & Northwest companion \\
\hline AM $2011-740 \ldots \ldots$ & 1995 Aug 24 & 5 & 600 & \\
\hline AM2030-303 ..... & 1995 Aug 23 & 4 & 600 & West companion \\
\hline 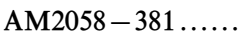 & 1995 Aug 23 & 5 & 600 & \\
\hline AM2100-381 ..... & 1995 Aug 25 & 5 & 600 & East companion \\
\hline AM $2105-332 \ldots \ldots$ & 1995 Aug 23 & 4 & 600 & \\
\hline AM2229-735 ..... & 1995 Aug 24 & 5 & 600 & \\
\hline AM $2238-575 \ldots \ldots$ & 1995 Aug 24 & 5 & 600 & \\
\hline AM2302-502 ..... & 1995 Aug 24 & 3 & 600 & Southwest companion \\
\hline AM2304-490..... & 1995 Aug 25 & 5 & 600 & \\
\hline AM2306-721 ..... & 1994 Oct 02 & 4 & 600 & \\
\hline AM2322-821 ..... & 1995 Aug 24 & 3 & 600 & \\
\hline AM $2330-451 \ldots \ldots$ & 1995 Aug 25 & 5 & 600 & \\
\hline AM2359-275 ..... & 1995 Aug 25 & 5 & 600 & \\
\hline
\end{tabular}

selection we rejected galaxies with angular diameter greater than $1^{\prime}$ because there is already photometric information for such galaxies in the Surface Photometry Catalog of ESOUppsala galaxies (Lauberts \& Valentijn 1989). In addition, we want to carry out accurate surface brightness photometry of the selected galaxies, including the faint outer extensions.

A total of 49 systems were observed that fulfill the above requirements, 31 that belong to AMC category 1 , seven that belong to category 9, and 11 apparent pairs (category 8). According to AMC we can summarize their characteristics as follows:

Category 1 (galaxies with interacting companions).-For each galaxy there is a smaller galaxy close by that either appears to be causing a perturbation or is itself distorted.

Category $1 a$ (strong interaction with spiral arms).-The spirals have well-defined arms so that the effect of the interaction in the arms is easily seen.

Category $1 b$ (strong interactions with main body).-All galaxies show companions strongly interacting with the main body of the larger galaxy. In most cases tidal disturbances on both galaxies can be observed.

Category 1c (wider interactions).--In this subcategory, the main galaxy is generally strongly disturbed, although the companion tends to be at a relatively great distance.

Category 1d (less strong interactions).- - The galaxies often are more widely separated than in previous subcategories. Such pairs are transition cases to category 8 (galaxies with apparent companions). 

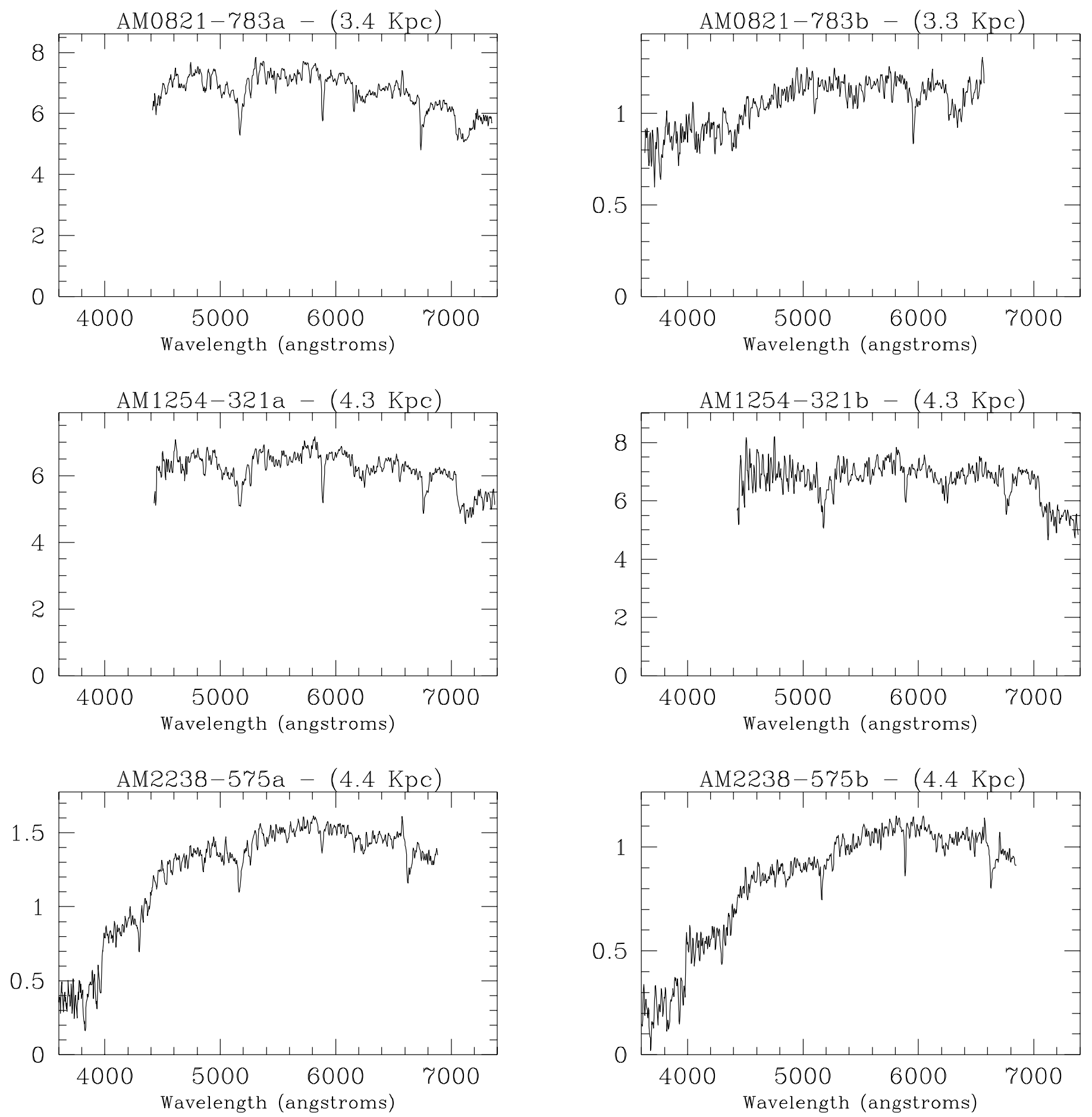

FIG. 1.-Individual spectra of the physical pairs. The plots show flux in units of $10^{-15} \mathrm{ergs} \mathrm{cm}^{-2} \mathrm{~s}^{-1} \AA^{-1}$ vs. wavelength in angstroms. The size of the region extracted is also specified on each spectrum.

Category 8 (galaxies with interacting companions).--In most cases in this category there are disturbances or peculiarities in either the companion or the main galaxy that support the idea that the pair is associated.

Category 9 (M51 types).- Galaxies that show a companion attached to spiral arms. They are classified according to the relative size of the companion, the openness of the spiral arm on which the companion appears, the type of central spiral, and the type of companion galaxy.
Observed galaxies in our sample are listed in Table 1. We specify the name and category number from AMC, right ascension, declination, morphological type, apparent $B$ magnitude, radial velocity, and blue extinction, the last taken from the Third Reference Catalog of Bright Galaxies (de Vaucouleurs et al. 1991, hereafter RC3). For ambiguous pairs in AMC photographs, the referenced pair is also specified in the last column. Note that all reference data are given for the brightest component of the pair, hereafter identified 

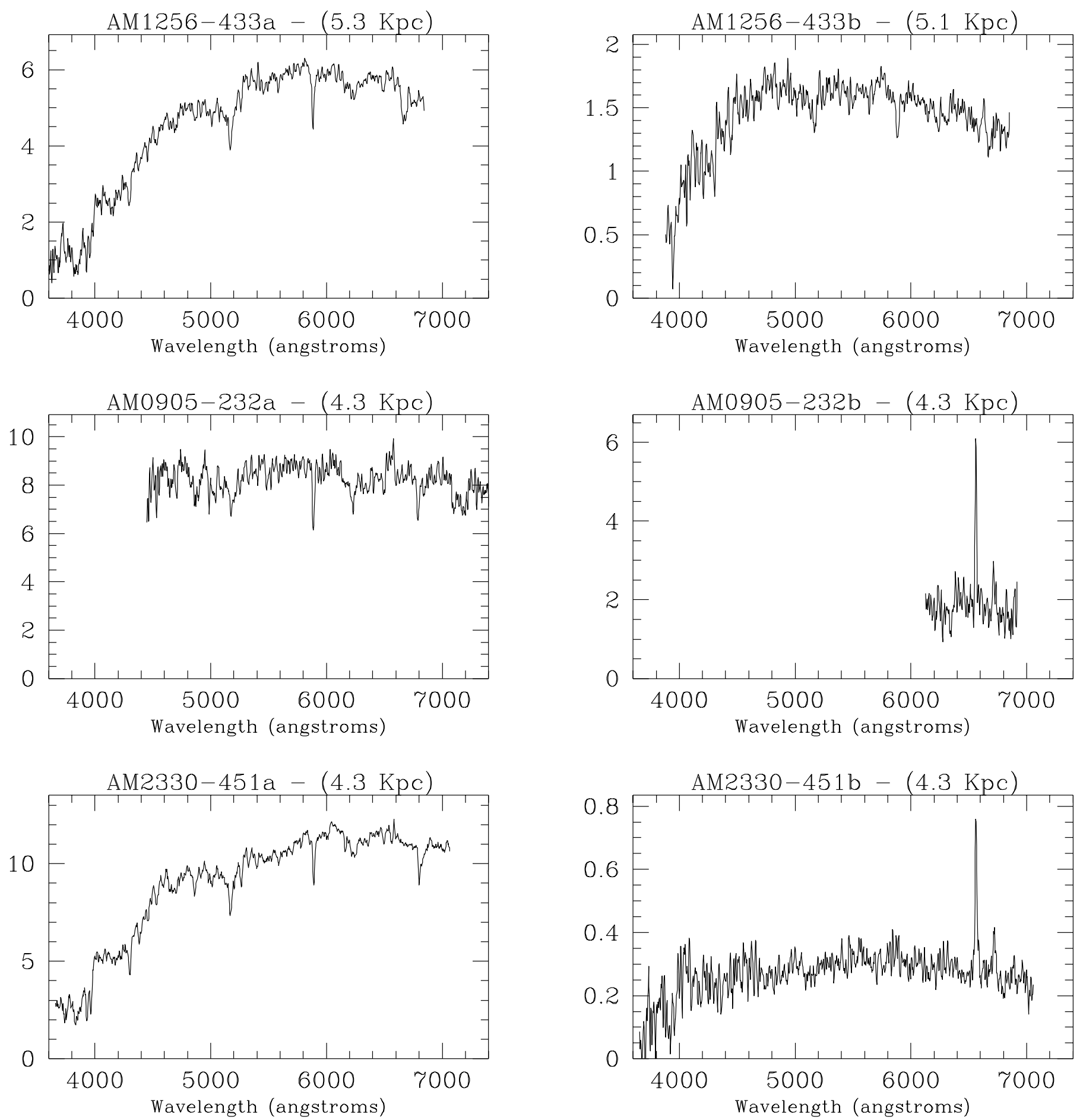

FIG. 1-Continued

as component $\mathrm{A}$. The darker object is hereafter referred to as component $\mathrm{B}$.

\section{OBSERVATIONS AND DATA REDUCTION}

Two-dimensional spectroscopy was carried out using the REOSC Spectrograph with a Tektronix $1024 \times 1024$ CCD at the $2.15 \mathrm{~m}$ Ritchey-Chrétien telescope at the Complejo Astronómico El Leoncito (CASLEO), San Juan, Argentina, over a total of nine nights in 1994 and 1995. The galaxies were observed using a slit 2.5 long and 3" wide. Spectra were obtained in two different wavelength ranges, 4500 $7400 \AA$ for the 1994 observations and 3600-7000 $\AA$ for the 1995 runs. In both cases we used a 300 lines $\mathrm{mm}^{-1}$ grating, resulting in a spectroscopic resolution of $3.3 \AA$. We obtained a set of short (400-600 s) exposures for each galaxy. In most cases the spectrograph slit was oriented so as to include both nuclei. In Table 2 we list the observation data and exposure time for each pair. For those galaxies with more than one companion, the observed galaxy is specified in the last column as component B. 

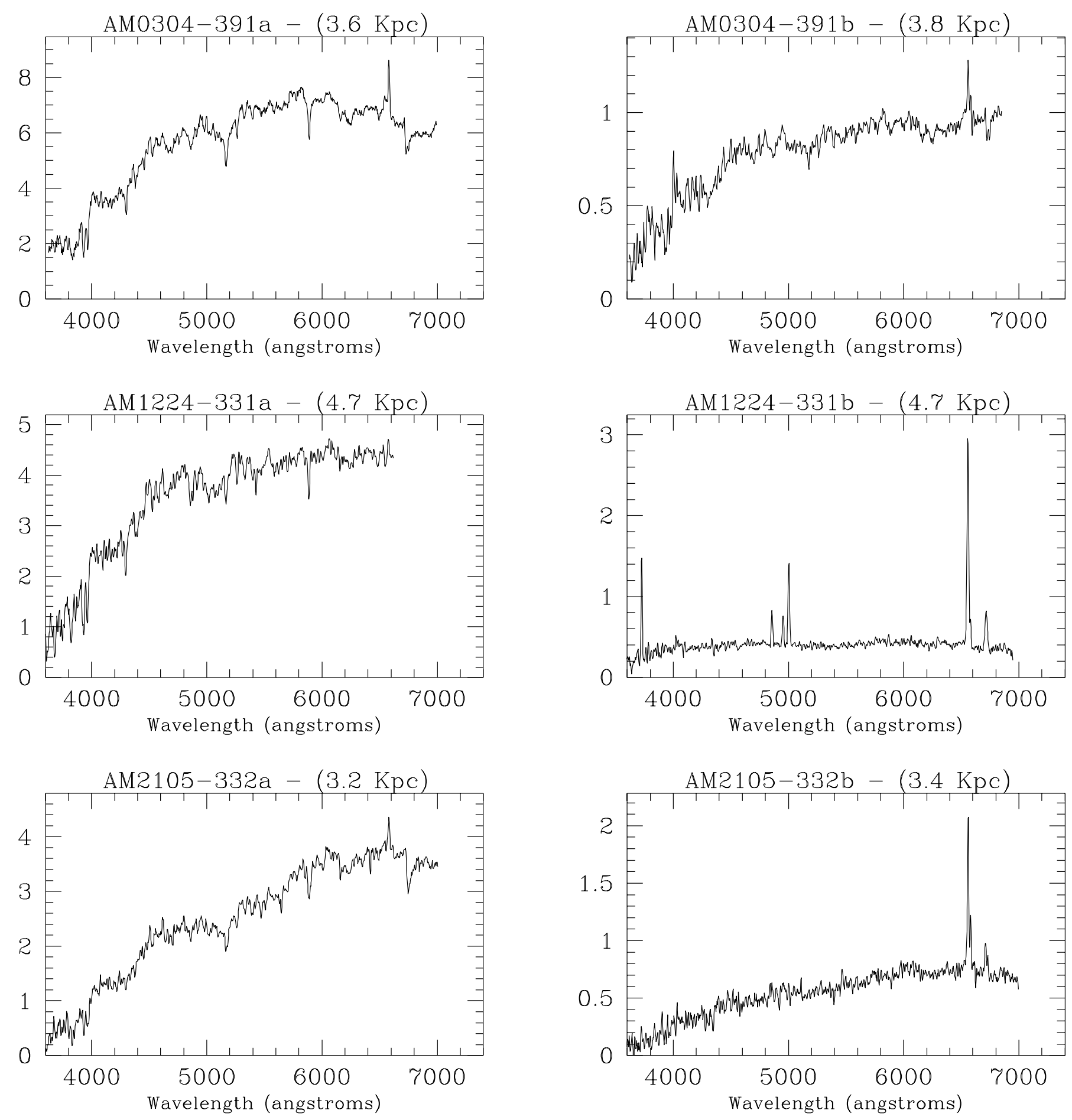

FIG. 1-Continued

All the spectra reduction was carried out using a Sun SPARCstation in the IRAF environment (developed by NOAO) at Cordoba Observatory, Argentina, and at the Instituto de Física, Universidade de Federal do Rio Grande de Sul, Porto Alegre, Brazil.

Each spectrum of a nucleus was extracted from a rectangular aperture centered at the peak of the brightness profile, and the corresponding scale over the region of the nucleus varies from 3 to $6 \mathrm{kpc}$. Throughout this paper we have assumed $H_{0}=75 \mathrm{~km} \mathrm{~s}^{-1} \mathrm{Mpc}^{-1}$.
The spectra were wavelength-calibrated using $\mathrm{He}-\mathrm{Ne}-\mathrm{Ar}$ comparison spectra Calibration accuracy was tested using sky lines and cross-correlated using one of the spatial sections as a template. The rms error amounts to less than 15 $\mathrm{km} \mathrm{s}^{-1}$.

The spectra were flux-calibrated using at least four standard stars per night taken from the Catalog of Southern Spectrophotometric Standards (Stone \& Baldwin 1983). We estimate a $\mathrm{rms}$ uncertainty of $0.12 \mathrm{mag}$ for residuals in fitting the system-sensitivity function. 

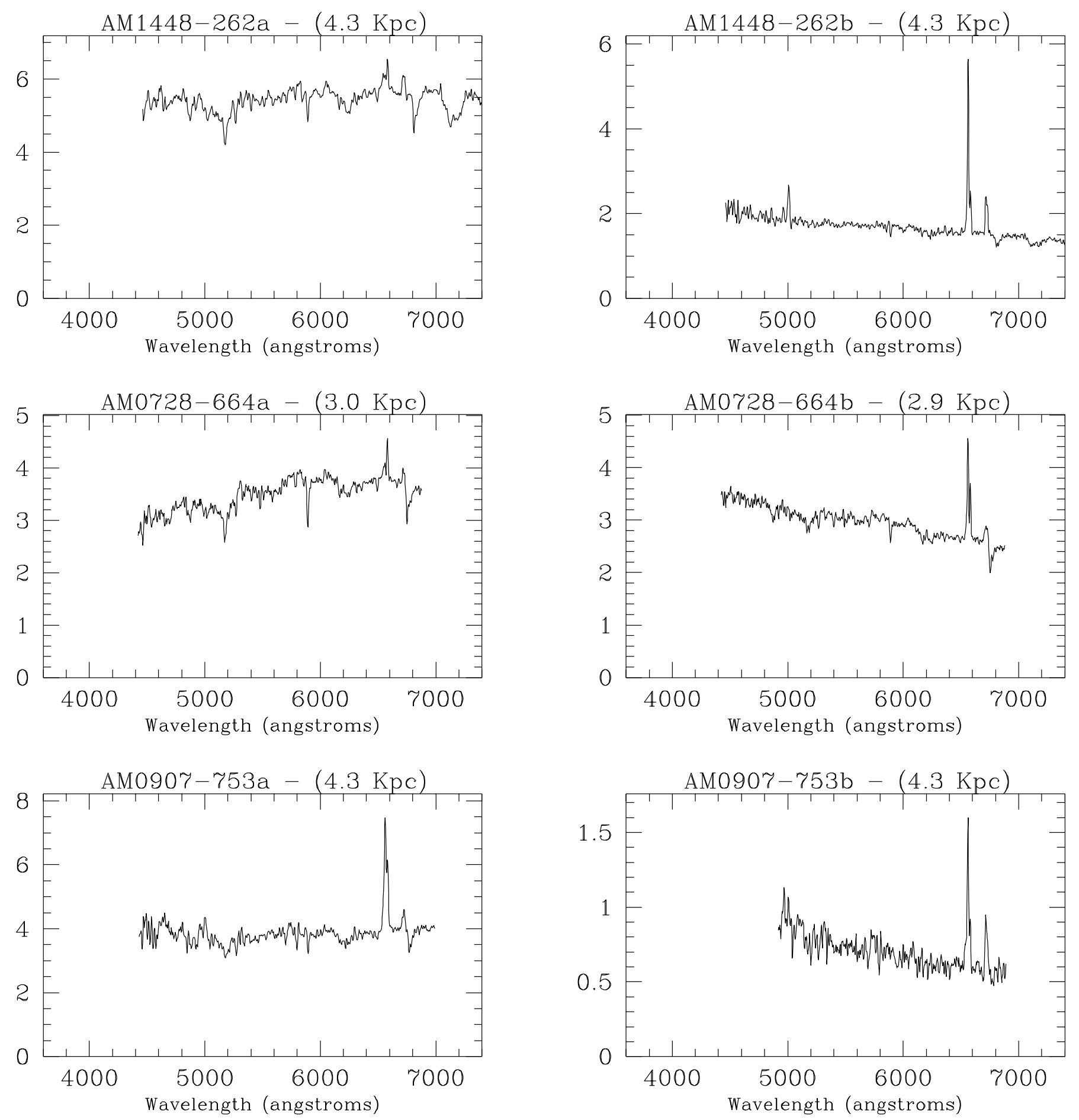

FIG. 1-Continued

We estimate that uncertainties in the EW measurements are typically $8 \%-12 \%$. However, for emission lines with EW less than $5 \AA$ the error amounts to $20 \%$.

The spectra have been corrected for galactic absorption using $A_{V}$ values taken from the RC3. For galaxies without such values we have interpolated values from neighboring galaxies in the RC3.

\section{RESULTS}

The spectra of all the galaxies in the sample are presented in Figures 1, 2, and 3. For Figures 1 and 2, component $A$ is displayed in the left panel while component B is displayed in the right panel. In the figures, wavelength is in angstroms and flux is in units of $10^{-15} \mathrm{ergs} \mathrm{cm} \mathrm{cm}^{-2} \mathrm{~s}^{-1} \AA^{-1}$. All spectra are redshift-corrected and plotted on a common wavelength scale of 3500-7400 $\AA$. The spectra have not been corrected for reddening within the galaxies themselves.

We have grouped the pairs in three categories according to their nature:

1. Physical pairs (Fig. 1). In this category we have included pairs with radial velocity differences between their components of less than $400 \mathrm{~km} \mathrm{~s}^{-1}$. This category is dis- 

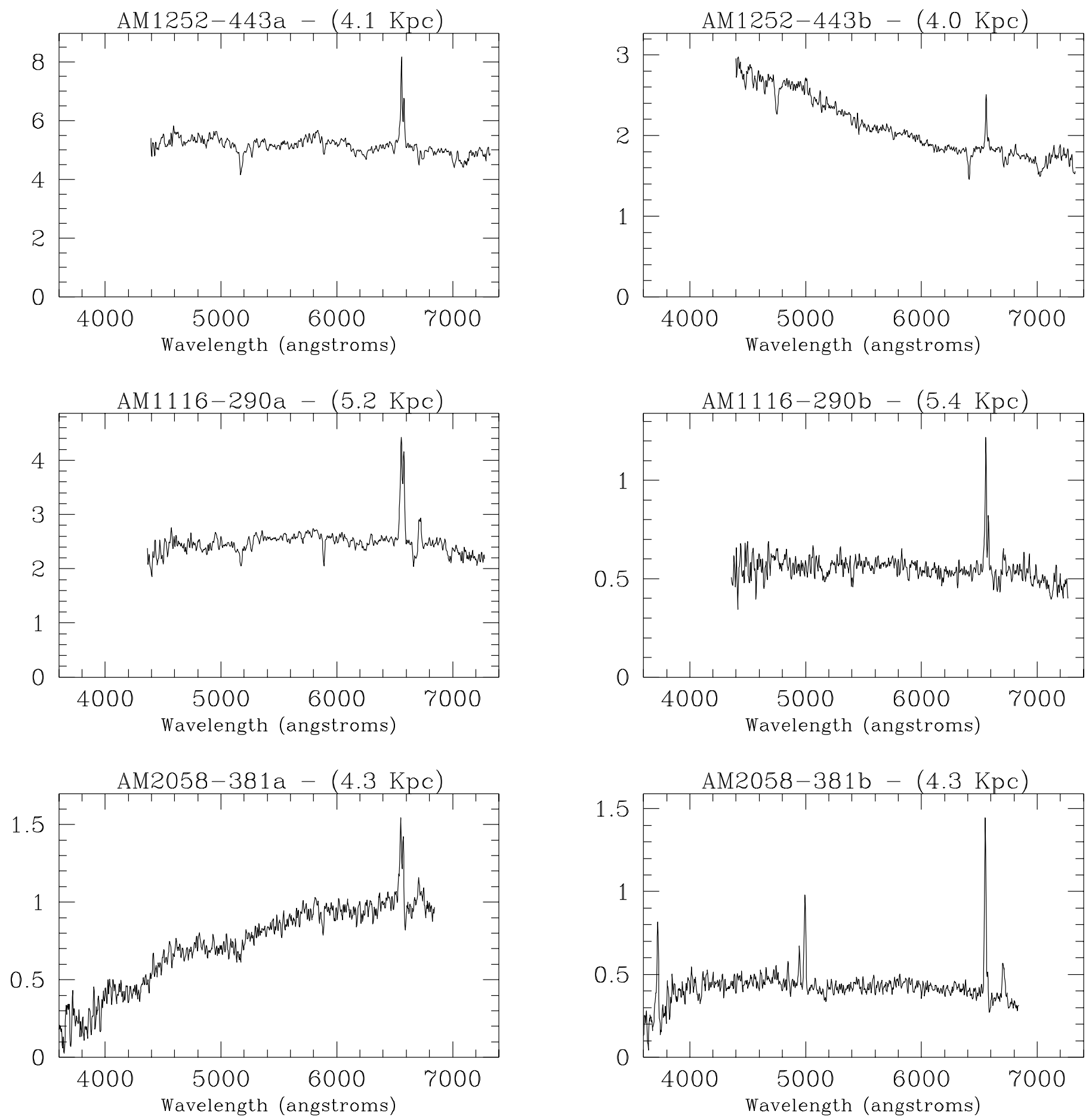

FIG. 1-Continued

cussed in more detail in $\S 5$.

2. Apparent pairs (Fig. 2). Radial velocity differences between the components are greater than $400 \mathrm{~km} \mathrm{~s}^{-1}$. These galaxies are AM 0407-612, AM 0550-342, AM 1208-273, AM 1921-534, AM 2100-381, AM 2302 - 502, and AM 2359-275.

3. Incomplete information (Fig. 3). For these pairs it was only possible to obtain the spectrum of component $\mathrm{A}$. We included here AM 0054-411, AM 0329-502, AM 0340-280, AM 1023-292, AM 1118-350, AM
1209-414, AM 1312-365, AM 1344-323, AM 2011 - 740, and AM 2304-490.

Radial velocities were measured from emission or absorption features using the cross-correlation technique developed by Tonry \& Davies (1979) as implemented in the $\mathrm{RV}$ program in the IRAF package. Typical rms values were around $75 \mathrm{~km} \mathrm{~s}^{-1}$. The values are listed in Table 3 . We found that four pairs (AM 0407-612, AM 0550-342, AM 1208-273, and AM 2100-381) are apparent pairs instead of physical ones, as can be inferred from their radial veloci- 

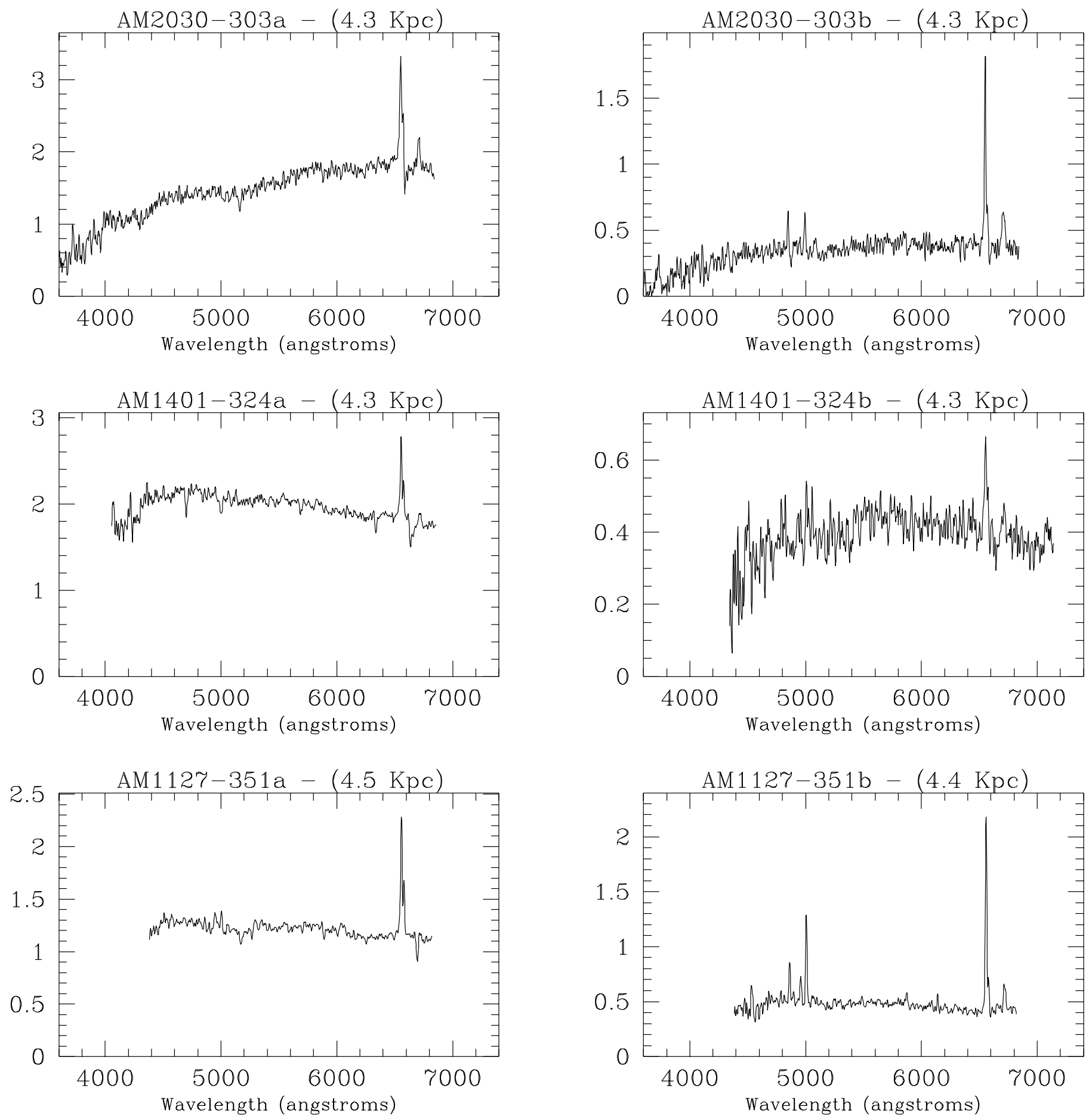

FIG. 1-Continued

ties. On the other hand, several pairs listed in AMC as galaxies with apparent companions are truly physical pairs. They are AM 0103-302, AM 0821-783, AM 1127-351, AM 1254-321, and AM 2238-575.

For all the spectra of the physical pairs we have also measured the EWs of the $\mathrm{H} \alpha+[\mathrm{N} \mathrm{II}] 6548$ and $6584 \AA$, $\mathrm{H} \beta$, and [O III] $5007 \AA$ emission lines in order to quantify the degree of activity in the nucleus and the star formation rate. These values are tabulated in Tables 4A and 4B and are discussed below.

\section{SPECTROSCOPIC CLASSIFICATION}

In this section we present a spectroscopic classification of the physical pairs (see $\S 4$ ) in four groups. This classification was made taking into account the type of emission-line spectrum observed in both components. In particular, $\mathrm{EW}(\mathrm{H} \alpha+[\mathrm{N}$ II) is used to measure the starburst activity of the galaxies (Kennicutt \& Kent 1983). The classification is as follows:

Group 1.-The spectra of both components are domi- 

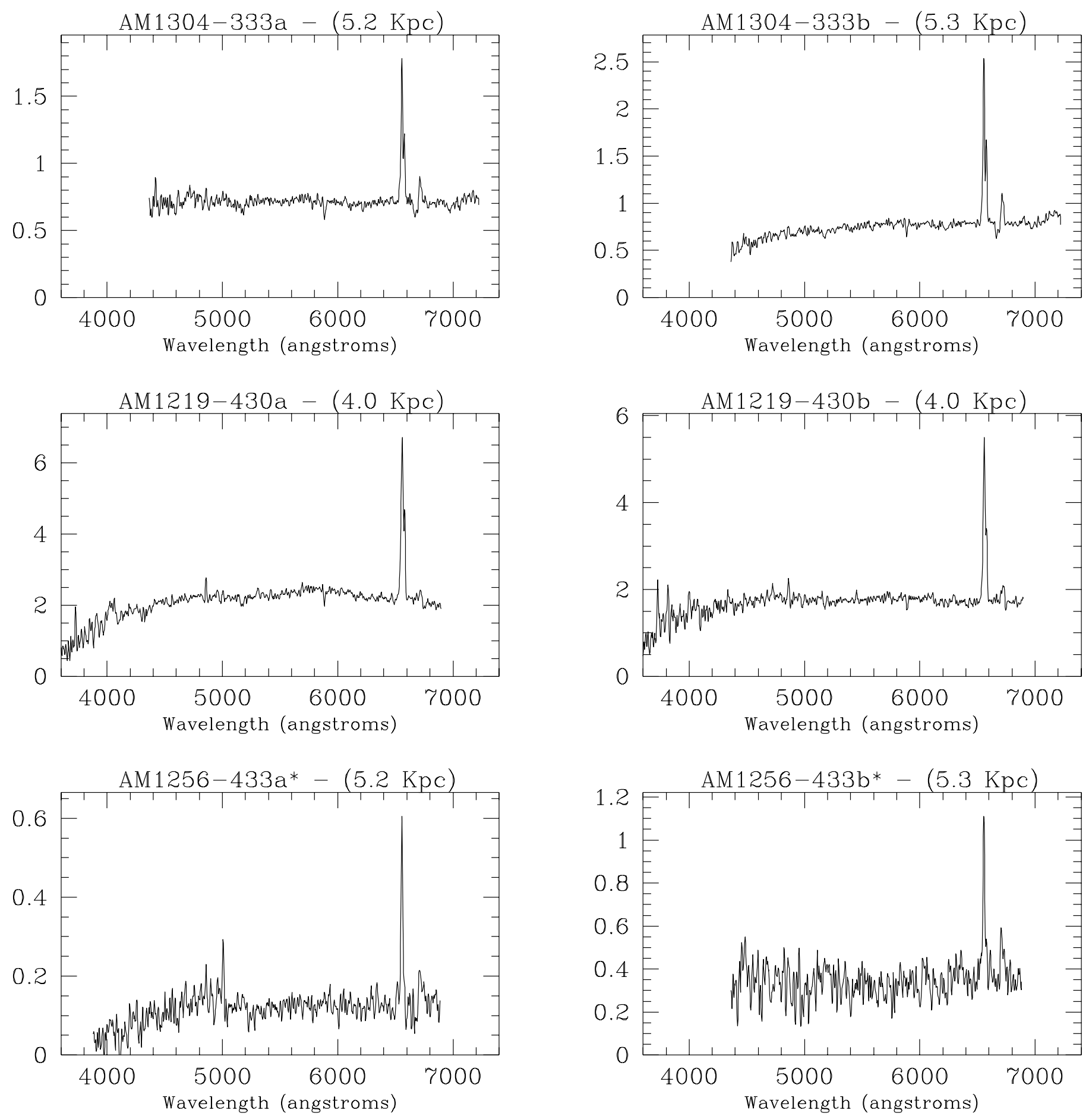

FIG. 1-Continued

nated by strong absorption features, e.g., TiO $6156 \AA$, Na $5890 \AA, \mathrm{Mg} \mathrm{I}+\mathrm{Mg} \mathrm{H} 5175 \AA$, the $G$ band of $\mathrm{CH} 4301 \AA$, CN $4200 \AA$, and Ca II $3933 \AA$. These obsorption features are typical of early-type galaxy spectra. No emission lines are observed. These galaxies are AM 0821-783, AM 1254-321, AM 2238-575 and AM 1256-433. The spectra of these galaxies are plotted in the first four rows of Figure 1.

Group 2.- The spectra of the A components show the same absorption features as in group 1, together with weak $\mathrm{H} \alpha+[\mathrm{N} \mathrm{II}]$ emission lines. The EW values of these lines are around $4 \AA$ and the $\mathrm{H} \alpha /[\mathrm{N}$ II] line intensity ratio is less than 1. The $B$ components have $H$ II region emission-type spectra with moderate excitation $(\mathrm{H} \alpha /[\mathrm{N}$ II $]>1)$. We include in this group the following galaxies: AM 0905-232, AM 2330-451, AM 0304-391, AM 1224-331, AM 2105-332, AM 1448-262, and AM 0728-664. The spectra are presented in the fifth through 11th rows of Figure 1.

Group 3.-The A components clearly show signatures of low-excitation H II-region emission-type spectra similar to those observed in the bulges of Sa-Sab galaxies. The 

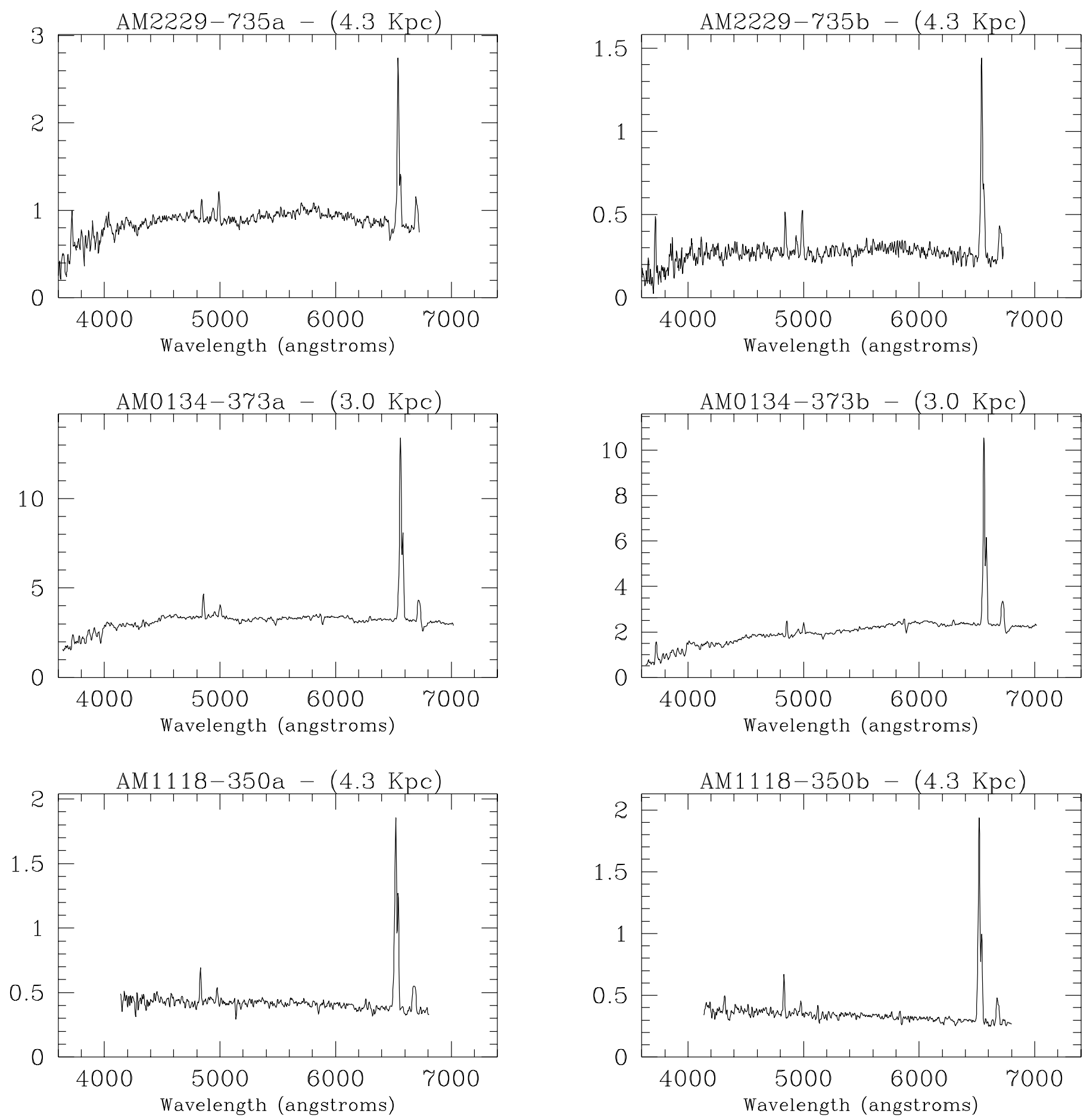

FIG. 1-Continued

absorption spectra for these galaxies have larger contributions from younger stellar populations than those observed for the A components in group 2. The B components have $\mathrm{H}$ II-region emission-type spectra with a wide range of excitation as inferred from the $\mathrm{EW}(\mathrm{H} \alpha+[\mathrm{N}$ II $])$ values, which range from 6 to $80 \AA$. The cases are AM 0907-753, AM 1252-433, AM 1116-290, AM 2058-381, AM 2030-303, AM 1401-324, and AM 1127-351. The spectra of these galaxies are plotted in the 12th through 18th rows of Figure 1.
Group 4.- Strong emission lines $(\mathrm{H} \alpha+[\mathrm{N} \mathrm{II}],[\mathrm{O}$ III], and $\mathrm{H} \beta$ ) are observed for the $A$ components indicating more excited spectra than in group 3. The observed $\mathrm{EW}(\mathrm{H} \alpha+[\mathrm{N} \mathrm{II}])$ have values greater than $30 \AA$. The B components also show $\mathrm{H}$ II-region emission-type spectra with very bright emission lines. The average of the $\mathrm{H} \alpha+$ [N II] equivalent widths for the B companions is $77 \AA$. This value is a factor of 2.6 larger than that reported for normal galaxies (Liu \& Kennicutt 1995, hereafter L\&K). Here we grouped AM 1304-333, AM 1219-430, AM 

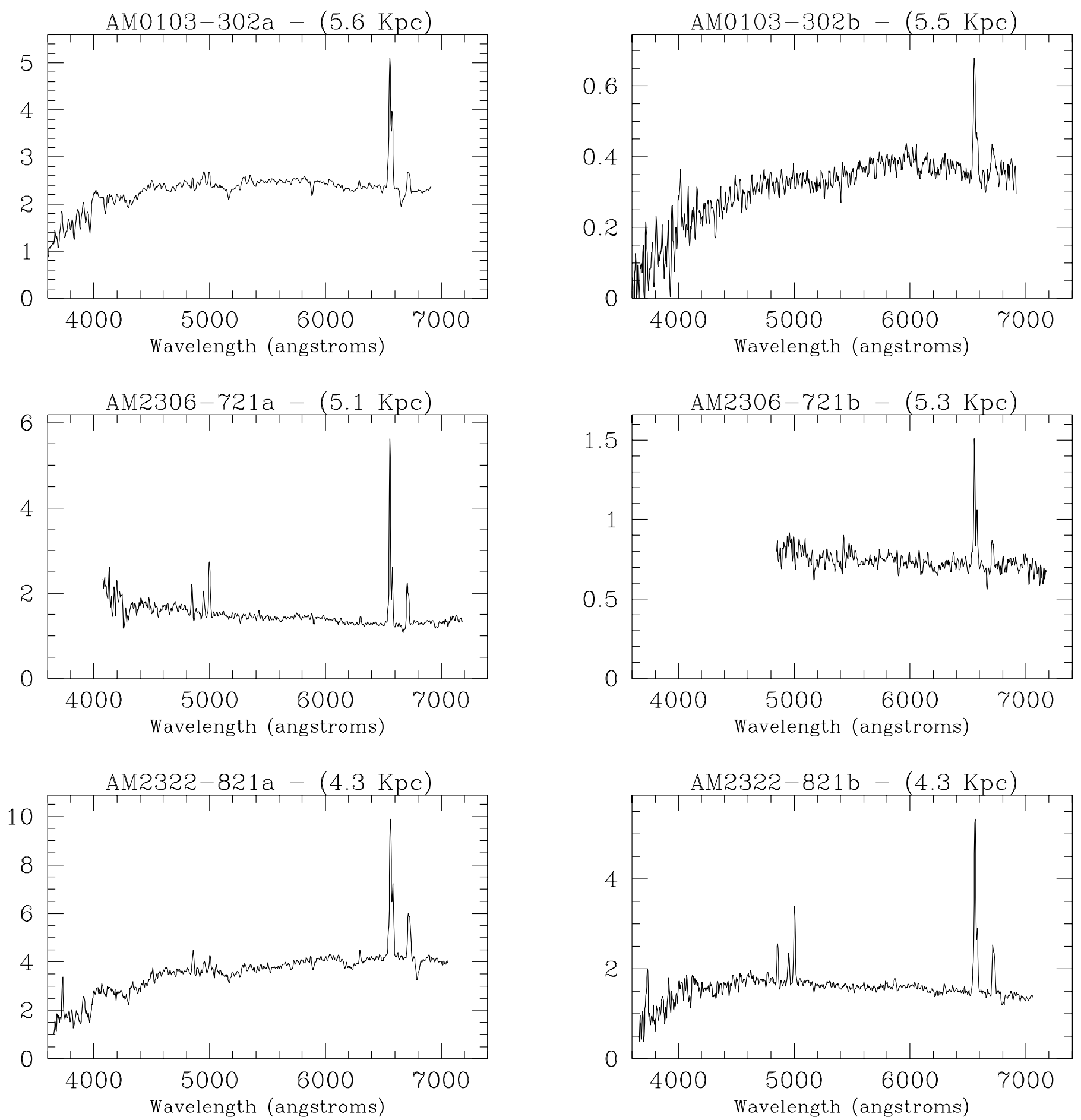

FIG. 1-Continued

1256-433, AM 2229-735, AM 0134-373, AM 1118-350, AM 0103-302, AM 2306-721, and AM $2322-821$. These spectra are plotted in the last nine rows of Figure 1.

Note the interesting fact that there is no evidence of Seyfert nuclei among the pairs, or even among the spectra of whole sample. This fact is discussed in more detail in $\S 6.3$.

In Tables 4A and 4B we list the galaxy pairs, ordered by groups, with tabulated values for the EWs of the emission lines described in $\S 4$. In Figures $4 a$ and $4 b$ we plot group members versus $\mathrm{EW}(\mathrm{H} \alpha+[\mathrm{N} \mathrm{II]})$. It is apparent in these figures that star formation increases with group number and that the B components show greater $\mathrm{EW}(\mathrm{H} \alpha+[\mathrm{N} \mathrm{II}])$ values than the A components.

It is also interesting to point out, as previously found by L\&K, that the morphological types of the interacting galaxies have a direct effect on the observed spectroscopic 

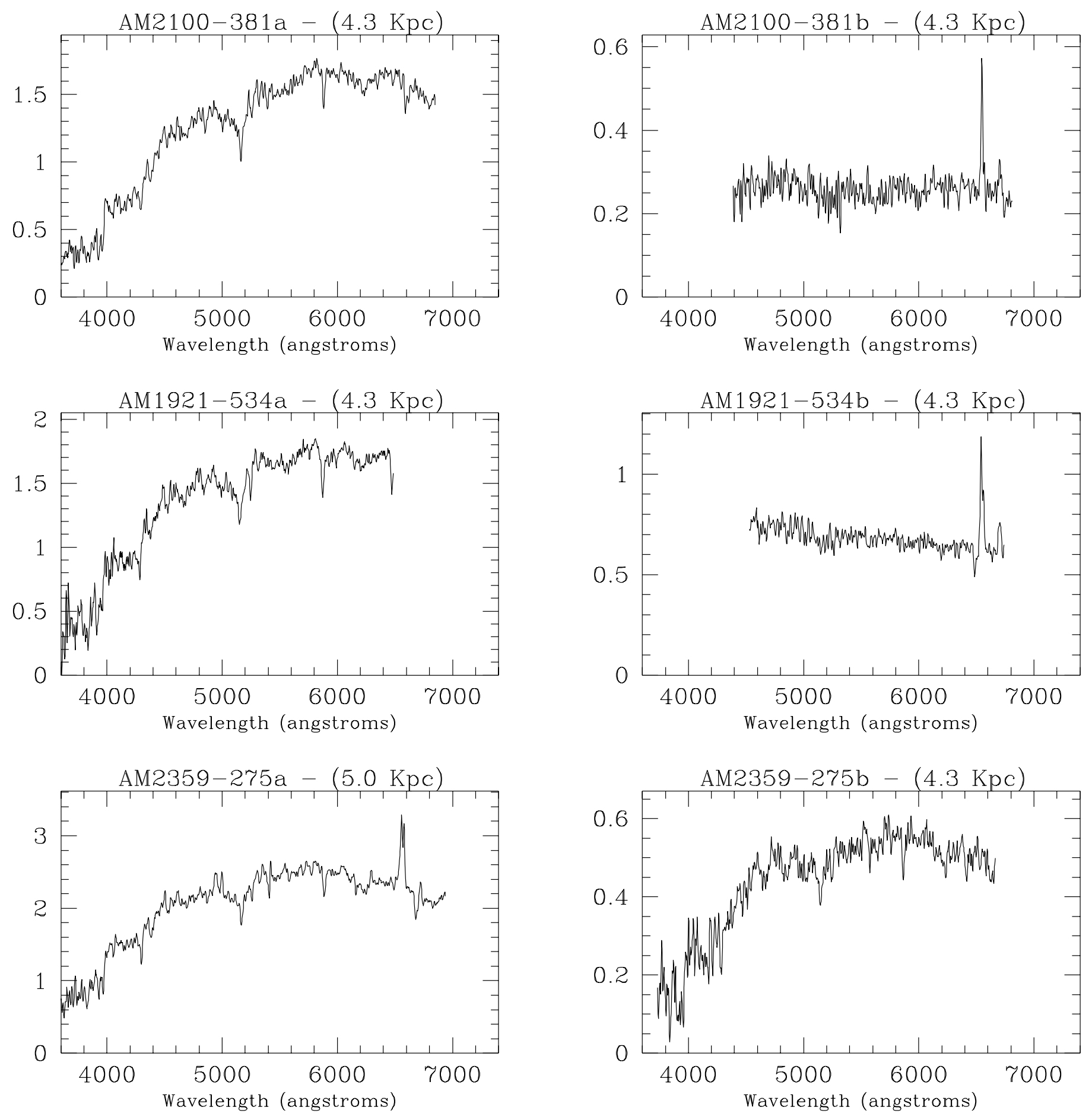

FIG. 2.-Same as Fig. 1, but for the spectra of the apparent pairs

characteristics. For example, early-type galaxies in group 1 do not show enhanced emission despite the interaction.

\section{SAMPLE PROPERTIES}

\subsection{Luminosity Distribution}

We have derived $M_{B}$ from the apparent integrated magnitudes estimated from CCD images of the pairs (Donzelli $\&$ Pastoriza 1997). The distances were calculated from radial velocities listed in Table 3 . Absolute magnitudes were corrected for galactic extinction using the values of Table 1 .

Figure $5 a$ shows the absolute magnitude distribution of the A components. It is clearly seen that these components are luminous spiral galaxies (see the morphological types in Table 1) with the luminosity distribution peak at $M_{B}=$ -21 . On the other hand, the peak of the luminosity function of the B components (Fig. $5 b$ ) is 2 mag fainter than that of the A components. From the AMC photographs it is noted that the B components spread over a wide range of morphological types. However, a more detailed analysis is needed to accurately determine the type of each galaxy. 

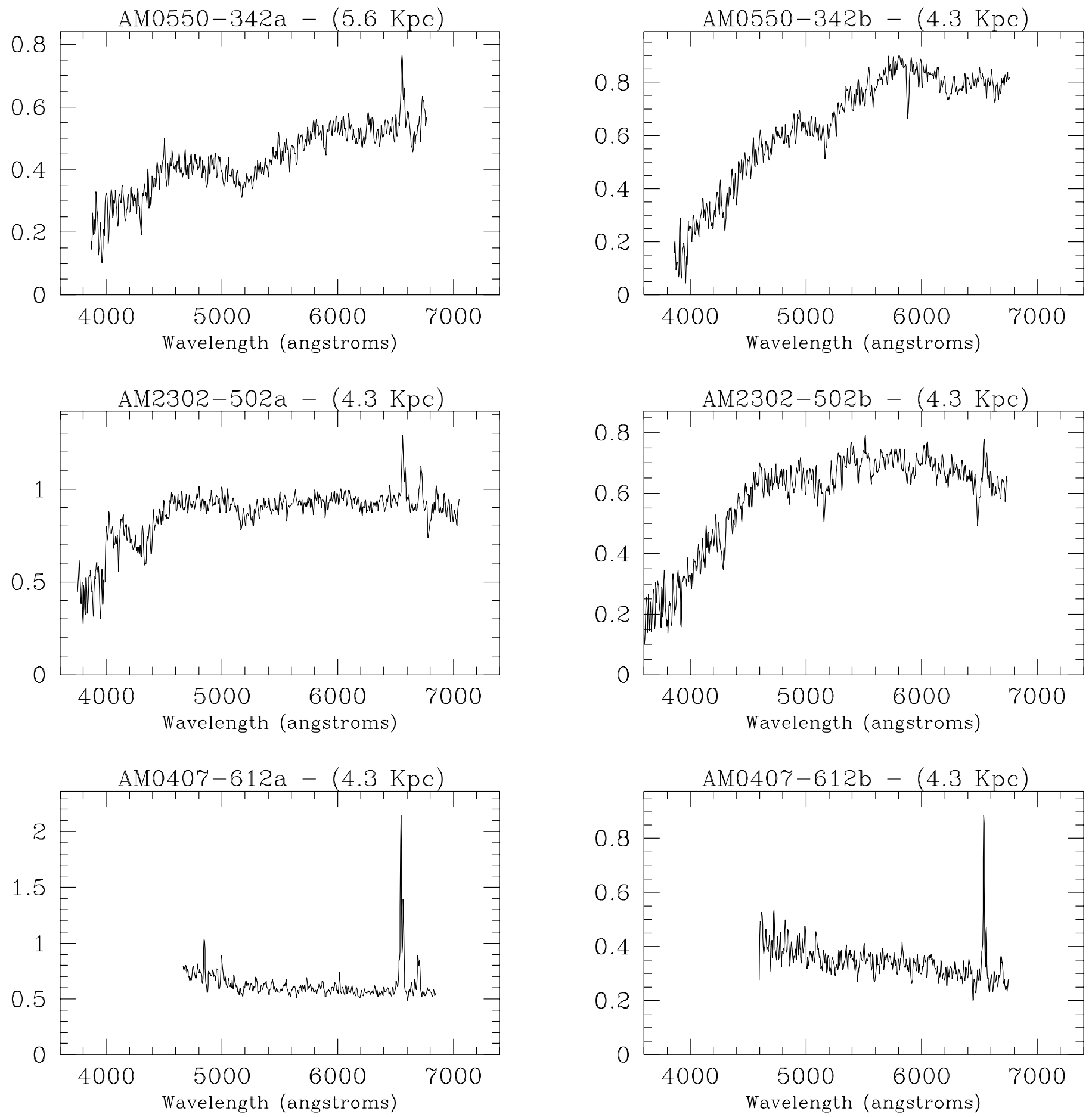

FIG. 2-Continued

\subsection{Star Formation Rates}

It is also interesting to compare the star formation events of these interacting galaxies with the star formation in field galaxies. We have performed this analysis through the distribution function of $\mathrm{EW}(\mathrm{H} \alpha+[\mathrm{N}$ II $])$, illustrated in Figures $6 a$ and $6 b$. Note that the A components show an average value of $\mathrm{EW}(\mathrm{H} \alpha+[\mathrm{N} I \mathrm{I}])=37 \AA$, slightly higher than that observed in normal isolated spiral galaxies, i.e., $29 \AA$ (Kennicutt \& Kent 1983). However, the mean $\mathrm{EW}(\mathrm{H} \alpha+[\mathrm{N} \mathrm{II}])$ for the B components is $54 \AA$, suggesting significantly enhanced SFRs compared to isolated galaxies, and even compared to the A components. This is an interesting result, since $\mathrm{S} \& \mathrm{~W}$ found, for a sample of interacting doubles of comparably sized galaxies, that the enhancement of the star formation activity is most likely to take place in both galaxies and has on average, the same strength. This effect can be explained by the models of Hernquist \& Mihos (1995), hereafter H\&M. They state that the structure of both primary and satellite galaxies is crucial for the generation of gas inflows and, therefore, star formation events. For 

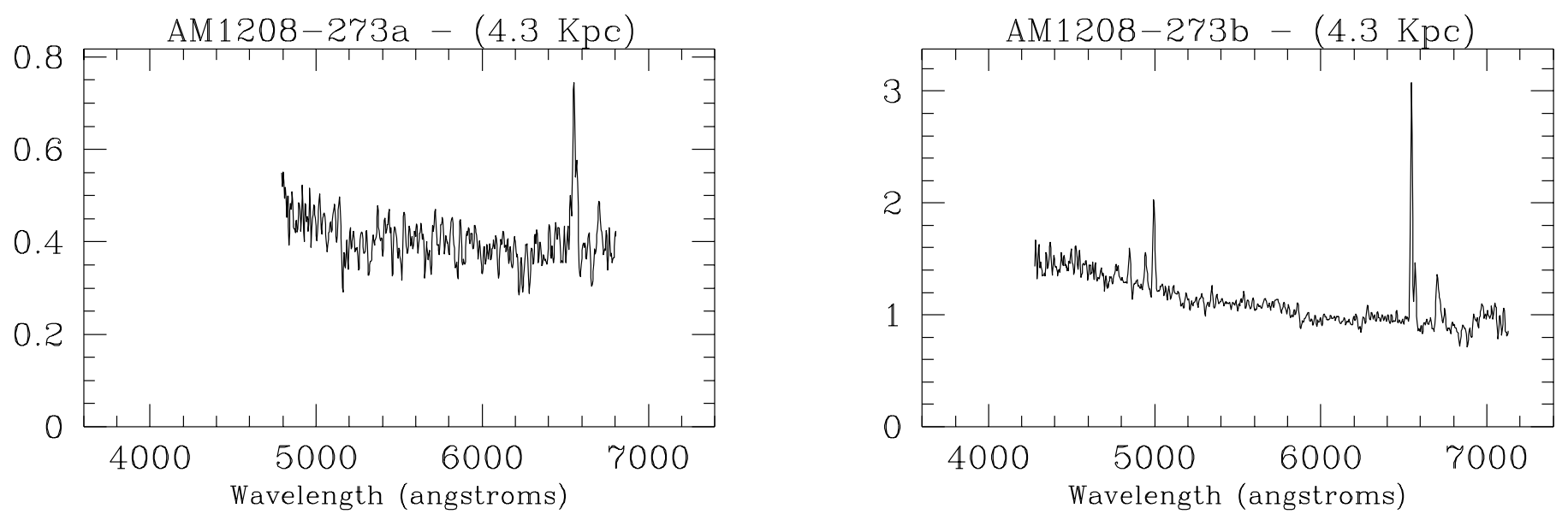

FIG. 2-Continued

instance, less dense satellites are easily torn apart by tidal forces. This effect avoids radial inflows of gas in the main component. On the other hand, although the effect on the secondary companion is not clear, the higher SFR observed can plausibly be blamed on the interaction. It is also interesting to point out that the excitation degrees of the emission spectra observed in the B components are comparable to those observed by L\&K in advanced mergers.

No significant correlation was found between $\mathrm{EW}(\mathrm{H} \alpha+[\mathrm{N} \mathrm{II}])$ and $M_{B}$, the EW and the projected distance (measured between the galaxy nucleus and normalized by the diameter of the component A), or the EW and the velocity difference of the pairs. This fact is indicative that the SFR does not depend on the luminosity of the galaxies or the form of interaction. However, some authors suggest correlation between the separation of the pair and the far-IR/B ratio (Jones $\&$ Stein 1989) and the separation and the $\mathrm{H} \alpha$ emission (Kennicutt et al. 1987).

\subsection{Interactions and Active Nuclei}

The effect of galaxy interactions on the appearance of AGN activity is a very delicate issue. Many papers (Keel et al. 1985; MacKenty 1989; Veilleux, Kim, \& Kron 1995, and references therein) show that Seyfert nuclei appear in interacting galaxies more frequently than in isolated galaxies, but many others (Dahari 1985; Bushouse 1986; S\&W; L\&K) have found a deficiency of AGNs among interacting or merging systems.

On the other hand, the enhancement of star formation activity observed in our sample can be understood through numerical simulations of mergers between gas-rich disks and less massive dwarf galaxies (H\&M). As a consequence of the interaction, the gas in the disk loses its angular momentum and is forced to fall into the inner regions. The radial inflows accumulate large fractions of gas in the nuclear regions of the disk and, given the high density of the nuclear gas, it is possible to identify these condensations with regions of intense star formation, like those reported in some active galaxies.

These models are consistent with the enhancement of the star formation activity observed in galaxies of groups 3 and 4 , since, as noted before, we observe in their nuclear regions
$\mathrm{EW}(\mathrm{H} \alpha+[\mathrm{N} \mathrm{II}])$ values larger than those observed in isolated galaxies.

On the other hand, if these models could reproduce the effects of the interactions in the sample galaxies of groups 3 and 4, we should expect, besides strong star formation events, nuclear activity, e.g., Seyfert 1 or Seyfert 2 nuclei among the observed pairs. As was pointed out in $\S 5$, there is a lack of Seyfert galaxies in our sample. One could argue that the lack of Seyfert-type nuclei is due to the fact that some of the galaxies of the sample could have strong circumnuclear star-forming regions (Pastoriza \& Donzelli 1997). Therefore, the H II-region spectra would dilute the AGN spectra when the observations are performed with a low spatial resolution, as in our case (see § 3).

There are several nearby galaxies that show strong circumnuclear star-forming regions within a central region of 2 Kpc (Sérsic \& Pastoriza 1965). Recently, long-slit observations performed with larger telescopes have shown that some of these galaxies, like NGC 1097, NGC 1672, and NGC 1365, contain LINERS or Seyfert 2 nuclei (Philips et al. 1983; Storchi-Bergmann, Baldwin, \& Wilson 1993, Storchi-Bergmann et al. 1995).

Therefore, the lack of AGNs among our galaxy sample as well as in S\&W's and L\&K's samples are not conclusive. High spatial resolution observations are needed in order to resolve the inner $1 \mathrm{kpc}$ of the galaxies.

\subsection{Ages of the Star Formation Events}

The star formation histories of the galaxies can be inferred through observable properties, such as the equivalent widths of the emission lines, $\mathrm{EW}(\mathrm{H} \alpha)$ and $\mathrm{EW}(\mathrm{H} \beta)$, and the ratio $[\mathrm{O}$ III] $/ \mathrm{H} \beta$ (Lequeux et al. 1981; Copetti, Pastoriza, \& Dottori 1986; Garcia Vargas \& Diaz 1994; Leitherer \& Heckman 1995, hereafter L\&H). It has been found that these parameters decrease monotonically as a function of time and consequently they are good star formation age indicators.

We have dated the star formation for our galaxy sample by comparing the equivalent width of the Balmer lines with the values taken from a grid of evolutionary synthesis models (L\&H). These models have been calculated for a population of massive stars using input parameters chosen 

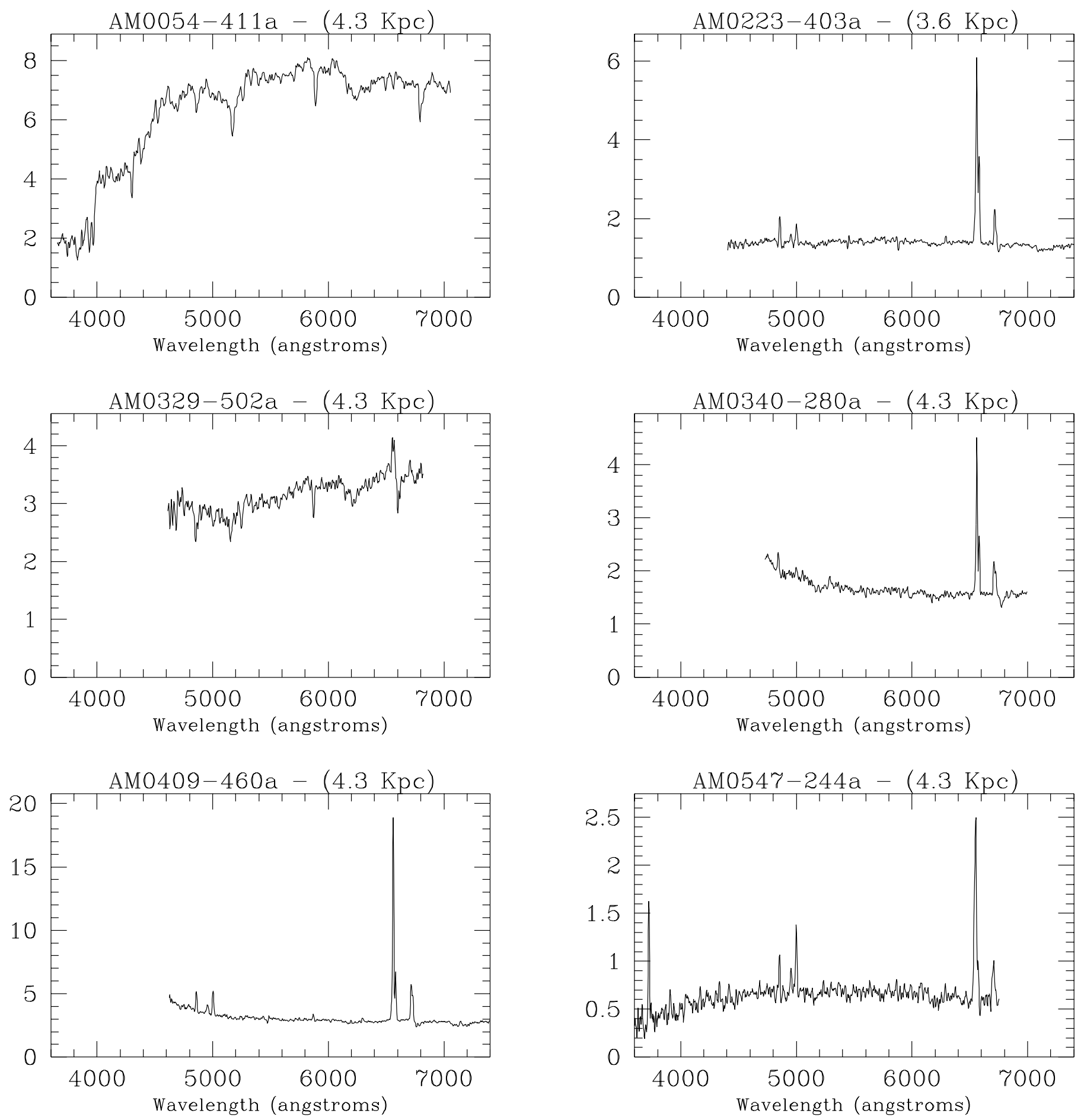

Fig. 3.-Same as Fig. 1, but for the spectra of the A components for which it was not possible to obtain the spectrum of its companion

to correspond to conditions typically found in objects such as $\mathrm{H}$ II regions, $\mathrm{H}$ in galaxies, blue compact dwarf galaxies, nuclear starburst galaxies, and infrared luminous starburst galaxies.

We have adopted, for most of the A and B components of groups 2 and 3, models with a constant star formation rate over a time interval of $300 \mathrm{Myr}$ with $\alpha=2.35, M_{\text {up }}=30$ $M_{\odot}$, and $Z=Z_{\odot}$. We make this choice because we observe, in the galaxy spectra, both emission and absorption lines, indicating that more than one star formation event has occurred in the lifetime of the galaxy. Therefore, this case can be approximated theoretically as a continuous star formation rate. It is interesting to point out that the values for an upper mass of $100 M_{\odot}$ do not fit the observed values.

For the B components in the above mentioned groups that show excited emission-region-type spectra, we have assumed the instantaneous star formation model $(\mathrm{L} \& \mathrm{H})$ because the $[\mathrm{O} \mathrm{III}] / \mathrm{H} \beta$ observed values are representative of an $\mathrm{H}$ II region younger than $10 \mathrm{Myr}$, according to the models of Copetti et al. (1986). These age values are in good 

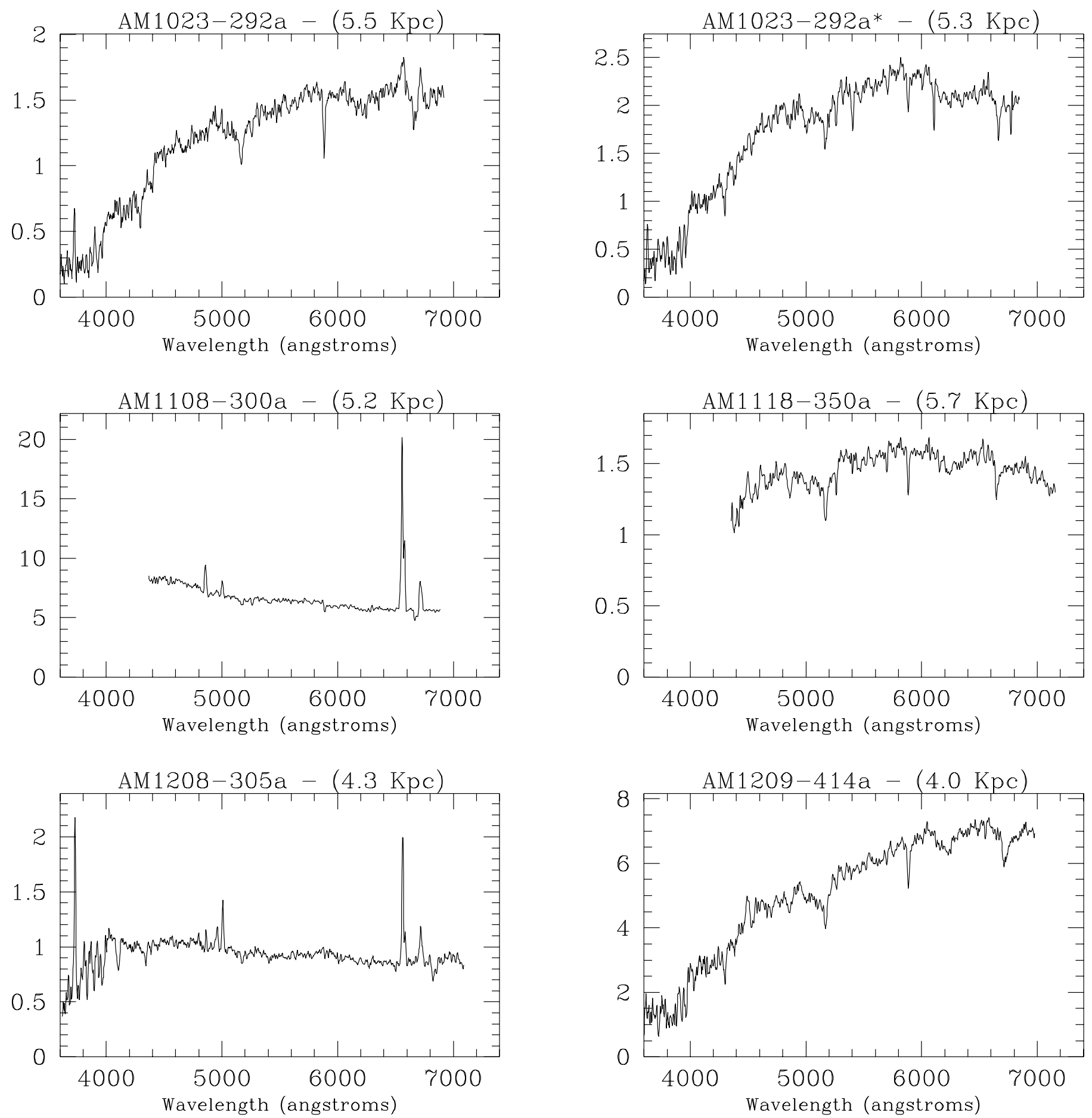

FIG. 3-Continued

agreement with those given for $\mathrm{EW}(\mathrm{H} \alpha)$ for the instantaneous star formation model of $\mathrm{L} \& \mathrm{H}$ with the same parameters as the constant star formation rate model. These B components are AM 1127-351, AM 1224-331, AM 1448 - 262, AM 2030 - 303, and AM 2058 - 381.

Based on the spectral type of group 4 galaxies, we have also adopted the criteria above in choosing the model to date star formation of those galaxies.

In Figure $5 a$ are plotted the observed $\mathrm{EW}(\mathrm{H} \alpha)$ values and the curve for the model of a continous star formation rate $\left(\alpha=2.35, M_{\text {up }}=30 M_{\odot}\right.$ and $\left.Z=Z_{\odot}\right)$, taken from L\&H. In this figure the A components are represented by stars, while the $\mathrm{B}$ components are indicated by squares.

On the other hand, Figure $5 b$ shows $\operatorname{EW}(\mathrm{H} \alpha)$ values for both $\mathrm{A}$ and $\mathrm{B}$ components, together with the curve that corresponds to the instantaneous burst from $\mathrm{L} \& \mathrm{H}$, with the same parameters and symbols as in Figure 7a.

From these figures, a first analysis shows that, on average, the star formation ages for the A components are comparable to those for the B components. We obtain an 

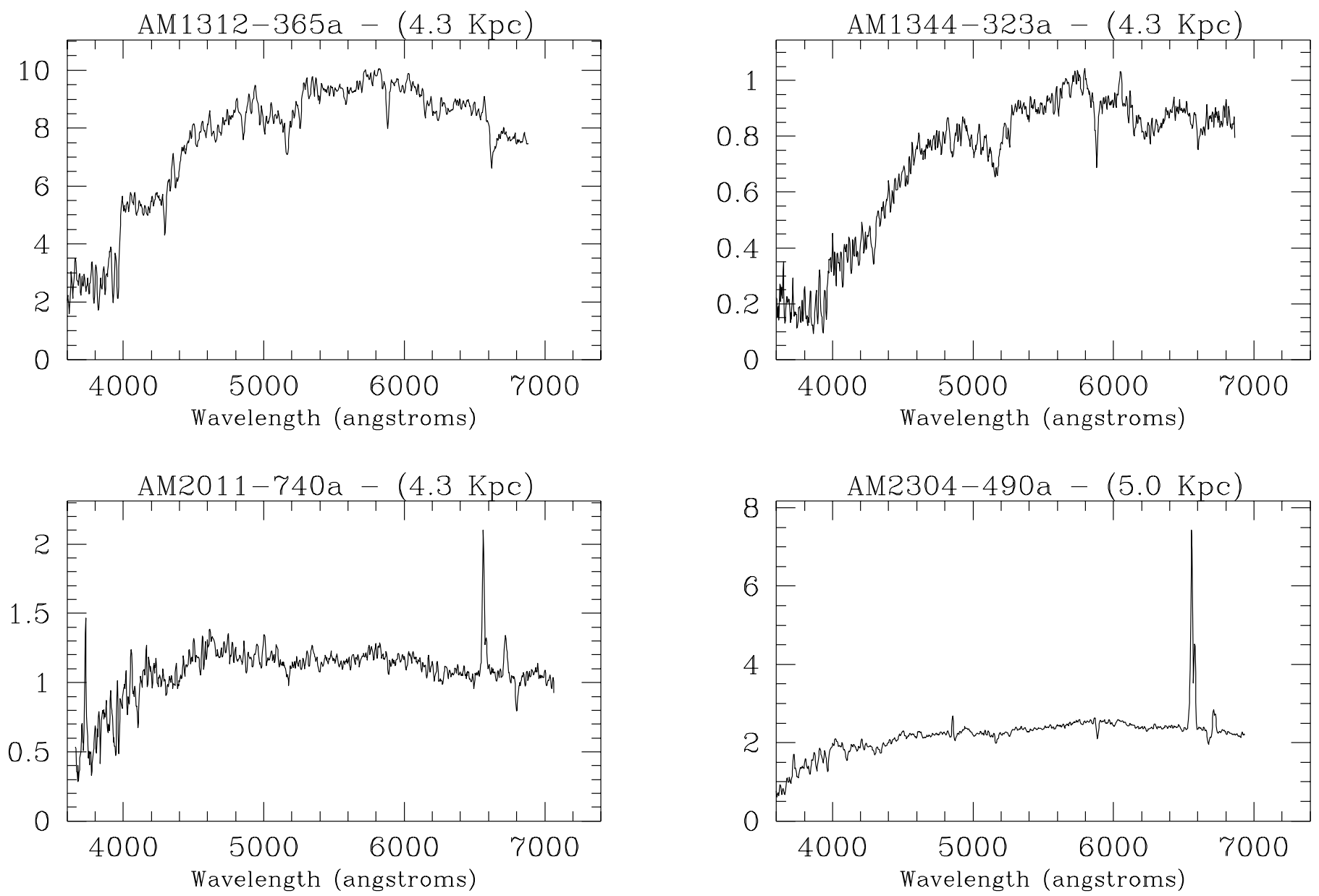

FIG. 3-Continued

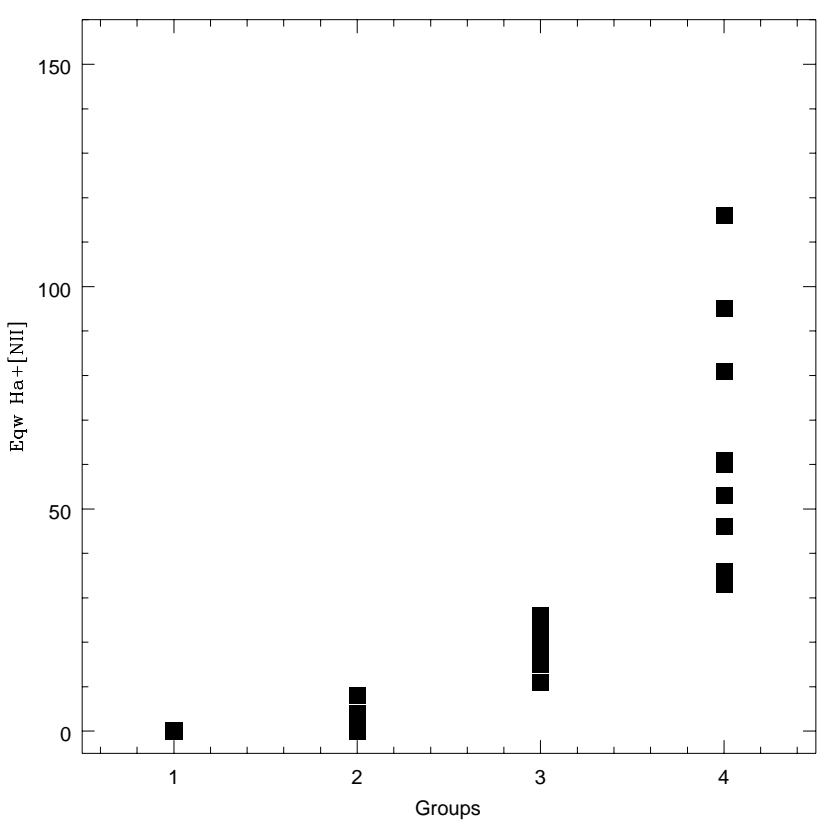

FIG. $4 a$

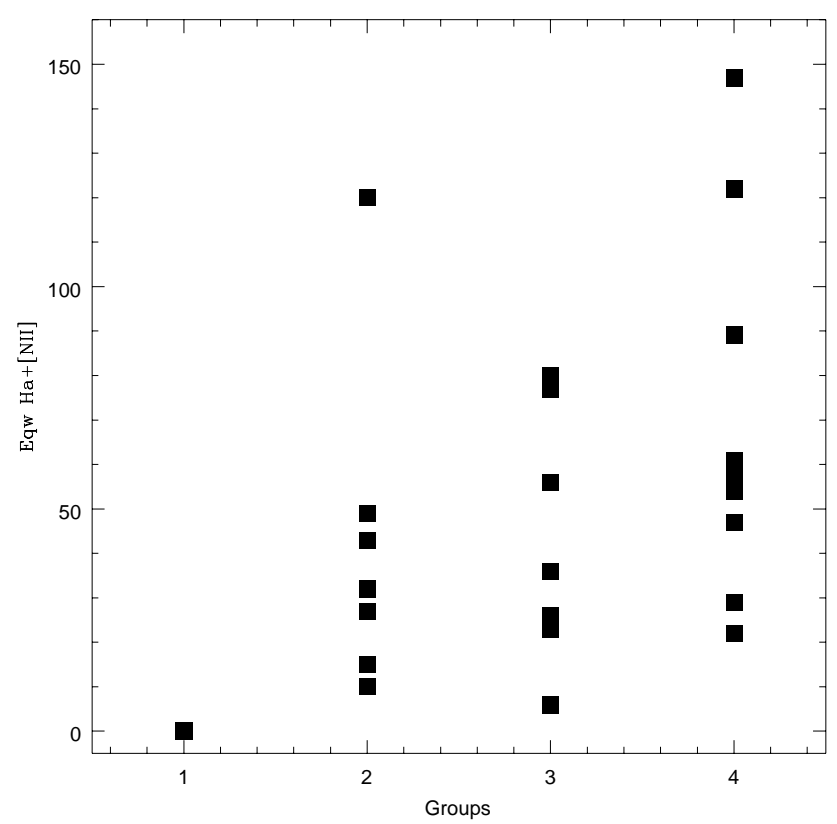

FIG. $4 b$

Fig. 4. $-(a) \mathrm{EW}(\mathrm{H} \alpha+\mathrm{N}$ II), in angstroms, vs. group number for the A components of the physical pairs. (b) Same as (a), but for the B components. 
TABLE 3

Measured Radial Velocities

\begin{tabular}{|c|c|c|}
\hline \multirow[b]{2}{*}{ NAME } & \multicolumn{2}{|c|}{$\begin{array}{c}c z \\
\left(\mathrm{~km} \mathrm{~s}^{-1}\right)\end{array}$} \\
\hline & A Component & B Component \\
\hline AM0054-411 ..... & 3501 & \\
\hline AM0103-302 ..... & 9617 & 9462 \\
\hline AM0134-373 ...... & 5137 & 5193 \\
\hline AM0223-403..... & 6216 & \\
\hline AM0304-391 ..... & 6288 & 6491 \\
\hline AM0329-502 ..... & 11793 & $\ldots$ \\
\hline 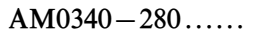 & 4401 & \\
\hline AM0407-612 ..... & 14444 & 18563 \\
\hline AM0409-460 ..... & 4951 & 4854 \\
\hline 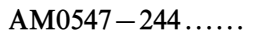 & 13164 & \\
\hline AM $0550-342 \ldots \ldots$ & 10293 & 9654 \\
\hline AM0728 $-664 \ldots \ldots$ & 5222 & 5055 \\
\hline AM0821-783..... & 5780 & 5679 \\
\hline AM0905-232 ..... & 3495 & 3492 \\
\hline AM0907-753 ..... & 4539 & 4786 \\
\hline AM1023-292 ..... & 9415 & $\ldots$ \\
\hline AM1023-292..... & 9175 & $\ldots$ \\
\hline 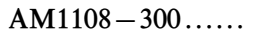 & 8890 & \\
\hline AM1116-290..... & 9042 & 9325 \\
\hline AM1118 $-350 \ldots \ldots$ & 9761 & 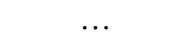 \\
\hline AM1118 $-350 \ldots \ldots$ & 24190 & 24071 \\
\hline AM1127-351 ..... & 7738 & 7629 \\
\hline AM1208-273 ..... & 14778 & 12433 \\
\hline AM1208-305...... & 2057 & $\ldots$ \\
\hline AM1209-414 ...... & 6869 & $\ldots$ \\
\hline AM1219-430 ..... & 6957 & 6879 \\
\hline AM1224-331 ..... & 8022 & 8173 \\
\hline AM1252-443..... & 7103 & 6838 \\
\hline AM1254-321 ..... & 4704 & 4825 \\
\hline AM1256-433 ...... & 9215 & 8811 \\
\hline AM1256-433..... & 9014 & 9183 \\
\hline AM1304-333 ..... & 9010 & 9091 \\
\hline AM $1312-365 \ldots \ldots$ & 10918 & $\ldots$ \\
\hline AM1344-323 ..... & 11669 & $\ldots$ \\
\hline AM1401 $-324 \ldots \ldots$ & 10321 & 10426 \\
\hline AM1448 $-262 \ldots \ldots$ & 2576 & 2738 \\
\hline AM1921 $-534 \ldots \ldots$ & 17515 & 16758 \\
\hline AM2011 $-740 \ldots \ldots$ & 3195 & $\ldots$ \\
\hline AM $2030-303 \ldots \ldots$ & 12465 & 12474 \\
\hline 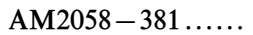 & 12383 & 12460 \\
\hline AM2100-381 ..... & 12314 & 13869 \\
\hline AM $2105-332 \ldots \ldots$ & 5449 & 5810 \\
\hline AM2229-735 ..... & 17535 & 17342 \\
\hline AM $2238-575 \ldots \ldots$ & 10823 & 10659 \\
\hline AM $2302-502 \ldots \ldots$ & 3784 & 16845 \\
\hline AM2304-490 ...... & 8634 & \\
\hline AM2306-721 ..... & 8762 & 9069 \\
\hline AM $2322-821 \ldots \ldots$ & 3680 & 3424 \\
\hline AM2330-451..... & 3189 & 3551 \\
\hline AM $2359-275 \ldots \ldots$ & 8534 & 20011 \\
\hline
\end{tabular}

age of $200 \mathrm{Myr}$ from the continuous star formation model and an age of $8 \mathrm{Myr}$ from the instantaneous burst model for both A and B components. However, a more detailed stellar population synthesis is needed to accurately date stellar formation events, as, for instance, using synthesized spectra. This analysis is beyond the scope of this paper.

\section{CONCLUSIONS}

We have presented in this paper individual spectra for 83 galaxies from a sample of 49 interacting galaxies pairs, from which we find that (1) 27 pairs are truly interacting galaxies, (2) seven are not physical pairs, and (3) for the remaining 15 pairs we do not have conclusive data to decide whether they are interacting galaxies.
TABLE 4A

A COMPONENTS

\begin{tabular}{cccc}
\hline \hline & \multicolumn{3}{c}{ EW $(\AA)$} \\
\cline { 2 - 4 } NAME & $\mathrm{H} \alpha+[\mathrm{N} \mathrm{II}]$ & $\mathrm{H} \beta$ & {$[\mathrm{O}$ III $]$} \\
\hline Group 1: & & & \\
AM0821-783 & $\ldots$ & $\ldots$ & $\ldots$ \\
AM1254-321 & $\ldots$ & $\ldots$ & $\ldots$ \\
AM2238-575 & $\ldots$ & $\ldots$ & $\ldots$ \\
AM1256-433 & $\ldots$ & $\ldots$ & $\ldots$ \\
Group 2: & & & \\
AM0905-232 & $\ldots$ & $\ldots$ & $\ldots$ \\
AM2330-451 & $\ldots$ & $\ldots$ & $\ldots$ \\
AM0304-391 & 8 & $\ldots$ & $\ldots$ \\
AM1224-331 & $\ldots$ & $\ldots$ & $\ldots$ \\
AM2105-332 & 3 & $\ldots$ & $\ldots$ \\
AM1448-262 & 4 & $\ldots$ & $\ldots$ \\
AM0728-664 & 4 & $\ldots$ & $\ldots$ \\
Group 3: & & & \\
AM0907-753 & 26 & $\ldots$ & 5 \\
AM1252-433 & 15 & $\ldots$ & $\ldots$ \\
AM1116-290 & 26 & $\ldots$ & 2 \\
AM2058-381 & 18 & $\ldots$ & $\ldots$ \\
AM1127-351 & 22 & $\ldots$ & 3 \\
AM2030-303 & 19 & $\ldots$ & $\ldots$ \\
AM1401-324 & 11 & $\ldots$ & $\ldots$ \\
Group 4: & & & $\ldots$ \\
AM1256-433 & 95 & 6 & 20 \\
AM1304-333 & 36 & $\ldots$ & 2 \\
AM1219-430 & 61 & 3 & 2 \\
AM2229-735 & 53 & 5 & 10 \\
AM0134-373 & 81 & 6 & 3 \\
AM1118-350 & 116 & 10 & 4 \\
AM0103-302 & 33 & $\ldots$ & 2 \\
AM2306-721 & 60 & 7 & 18 \\
AM2322-821 & 46 & 4 & 3 \\
\hline & & & \\
\hline
\end{tabular}

TABLE 4B

B COMPONENTS

\begin{tabular}{|c|c|c|c|}
\hline \multirow[b]{2}{*}{ NAME } & \multicolumn{3}{|c|}{ EW (̊̊) } \\
\hline & $\mathrm{H} \alpha+[\mathrm{N} \mathrm{II}]$ & $\mathrm{H} \beta$ & [O III] \\
\hline \multicolumn{4}{|l|}{ Group 1: } \\
\hline 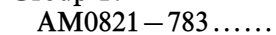 & $\ldots$ & $\ldots$ & $\ldots$ \\
\hline AM1254-321 $\ldots \ldots$ & $\ldots$ & $\ldots$ & $\ldots$ \\
\hline AM $2238-575 \ldots \ldots$ & $\ldots$ & $\ldots$ & $\ldots$ \\
\hline AM1256-433...... & $\begin{array}{l}\cdots \\
\cdots\end{array}$ & $\begin{array}{l}\cdots \\
\cdots\end{array}$ & $\begin{array}{l}\cdots \\
\cdots\end{array}$ \\
\hline \multicolumn{4}{|l|}{ Group 2: } \\
\hline AM0905-232 ..... & 27 & $\ldots$ & $\ldots$ \\
\hline 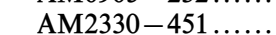 & 43 & $\ldots$ & $\ldots$ \\
\hline AM0304-391 ..... & 10 & $\ldots$ & $\ldots$ \\
\hline AM1224-331 .... & 120 & 18 & 35 \\
\hline AM $2105-332 \ldots \ldots$ & 32 & $\ldots$ & $\ldots$ \\
\hline AM1448 $-262 \ldots \ldots$ & 49 & 3 & 12 \\
\hline AM0728-664_..... & 15 & $\ldots$ & $\ldots$ \\
\hline \multicolumn{4}{|l|}{ Group 3: } \\
\hline AM0907-753 ..... & 36 & $\ldots$ & 2 \\
\hline AM1252-433..... & 6 & $\ldots$ & $\ldots$ \\
\hline AM1116-290..... & 26 & $\ldots$ & $\ldots$ \\
\hline 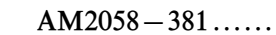 & 56 & 5 & 30 \\
\hline 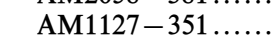 & 77 & 10 & 34 \\
\hline AM $2030-303 \ldots \ldots$ & 80 & 11 & 11 \\
\hline AM1401-324 ...... & 23 & $\ldots$ & 3 \\
\hline \multicolumn{4}{|l|}{ Group 4: } \\
\hline AM1256-433 ..... & 47 & $\ldots$ & $\ldots$ \\
\hline AM1304-333..... & 54 & $\ldots$ & $\ldots$ \\
\hline AM1219-430 ..... & 57 & 4 & 2 \\
\hline 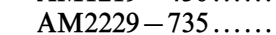 & 122 & 15 & 23 \\
\hline AM0134-373 ..... & 89 & 5 & 5 \\
\hline AM1118 $-350 \ldots \ldots$ & 147 & 15 & 5 \\
\hline AM0103-302 ..... & 29 & $\ldots$ & $\ldots$ \\
\hline 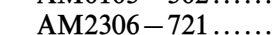 & 22 & $\ldots$ & $\ldots$ \\
\hline AM $2322-821 \ldots \ldots$ & 61 & $\dddot{9}$ & $\dddot{23}$ \\
\hline
\end{tabular}




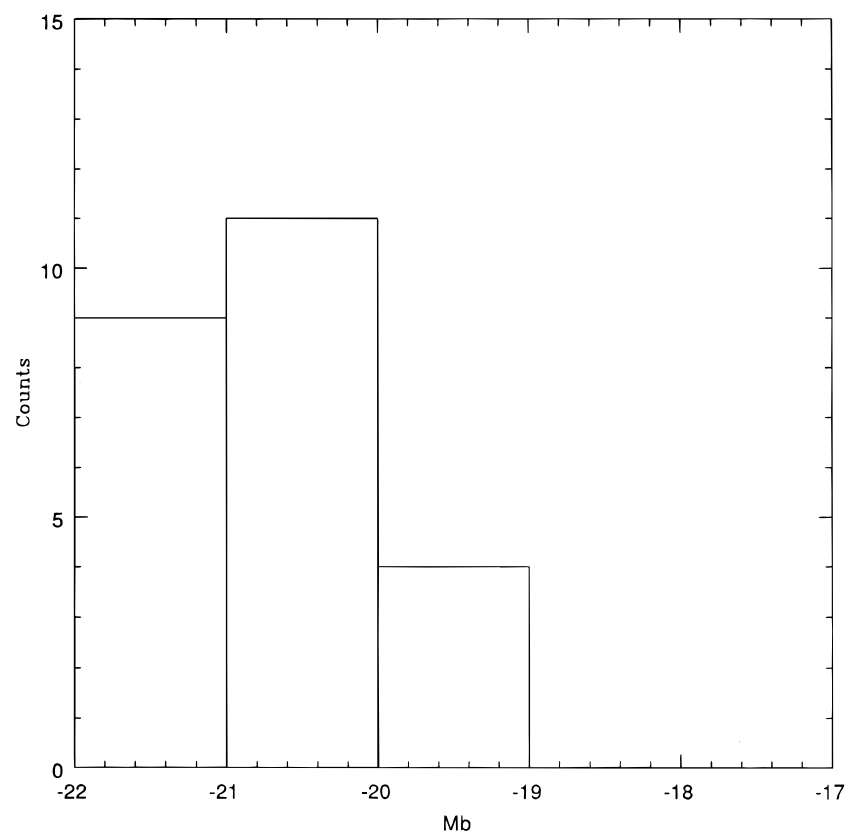

FIG. $5 a$

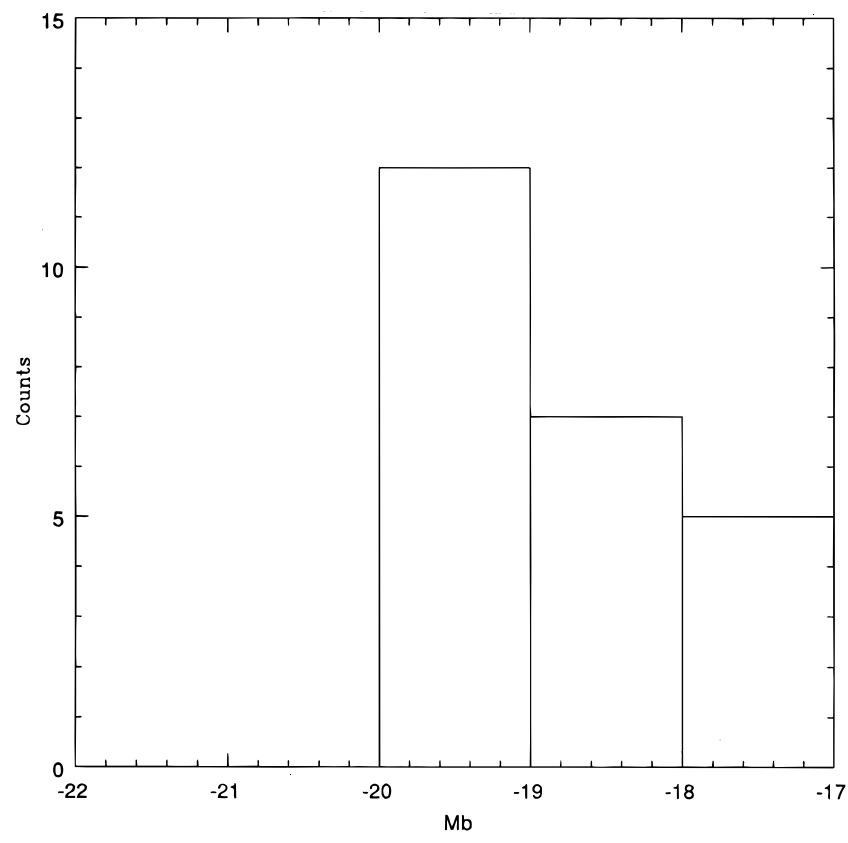

FIG. $5 b$

Fig. 5. (a) Absolute blue magnitude distribution for the A components of the physical pairs. (b) Same as (a), but for the B components.

From our data it can be seen that interactions produce a wide range of spectra, although the selected pairs were chosen irrespective of the morphological type of the galaxies.

This preliminary analysis of the data also confirms a characteristic of interacting galaxies already reported by other authors, that is, enhanced star formation rates (e.g., Bushouse 1987; Kennicutt et al. 1987). Moreover, in our data the strength of the star formation enhancement does not correlate with the type of interaction. Note also the fact that our sample of interacting systems has, on average,

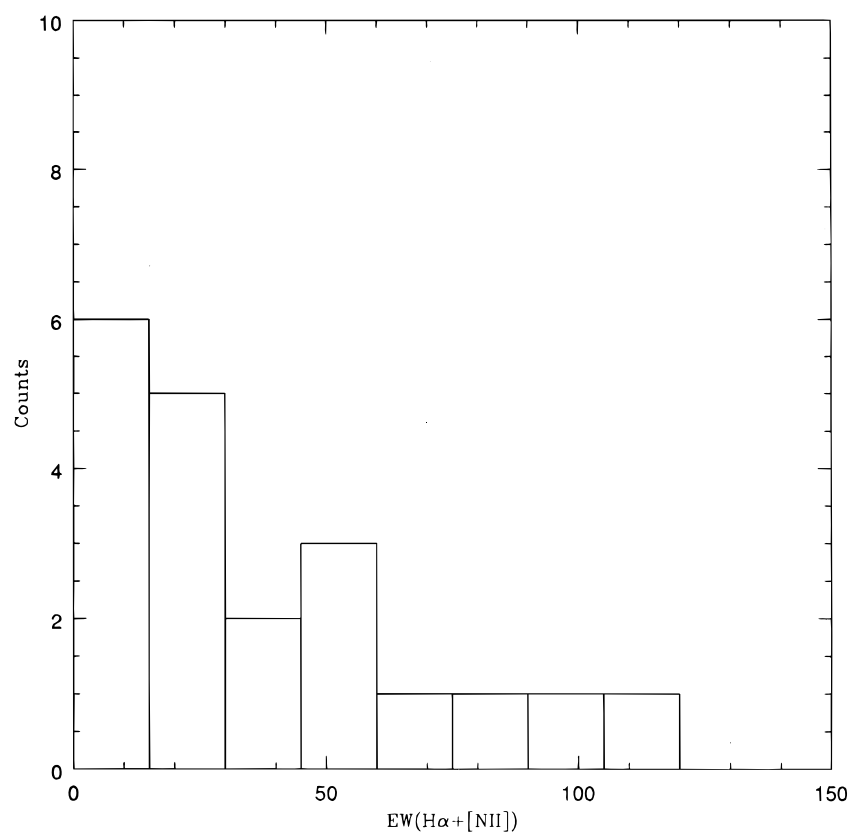

FIG. $6 a$
SFRs similar to those observed in well-advanced mergers, as can be inferred from $\mathrm{EW}(\mathrm{H} \alpha+[\mathrm{N} \mathrm{II}])$ values (L\&K).

On the other hand, although enhancement of star formation activity is more likely to take place at comparable strengths in each galaxy, for a sample of comparably sized galaxies (S\&W), for our case the enhancement is much stronger ( $\sim 50 \%$ on average) in the minor components of the pairs. One possible explanation of this effect is that the main galaxy could have a dense and massive bulge that inhibits the gas inflows in this component of the pair (H\&M).

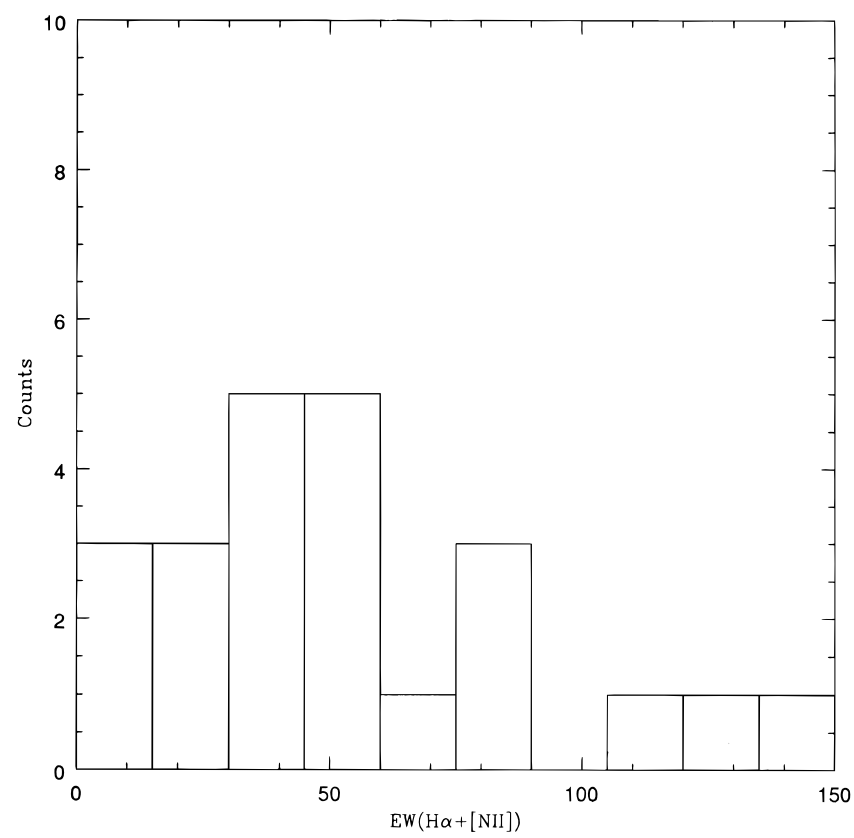

FIG. $6 b$

FIG. 6.-(a) Distribution function of $\mathrm{EW}(\mathrm{H} \alpha+[\mathrm{N} \mathrm{II}])$, in angstroms, for the A components of the physical pairs. (b) Same as (a), but for the B components. 


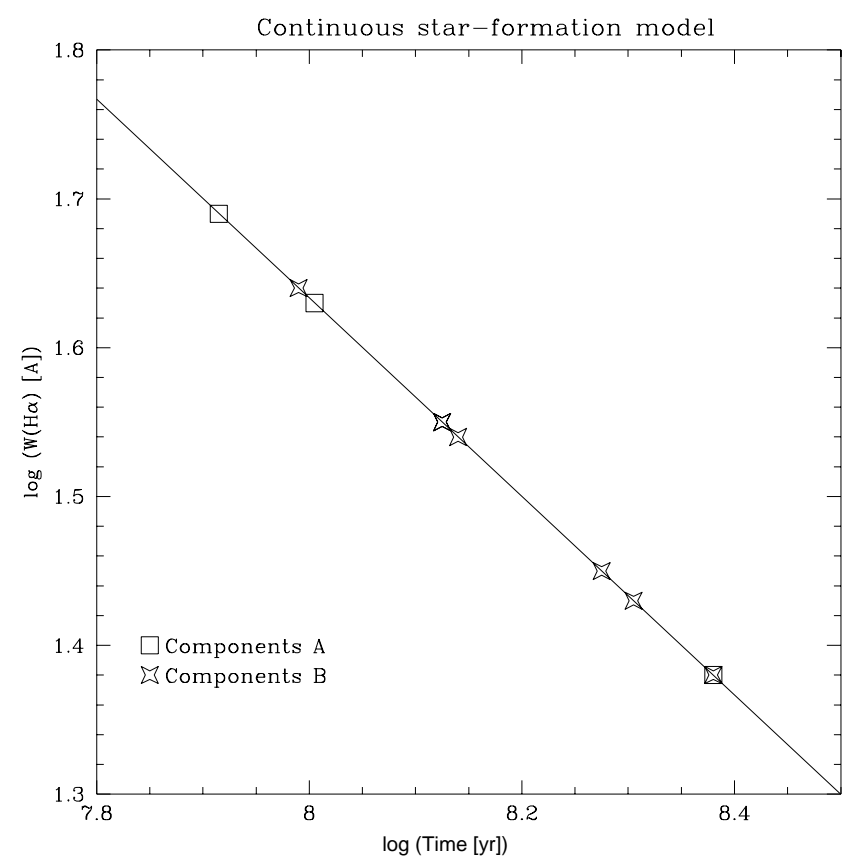

FIG. $7 a$

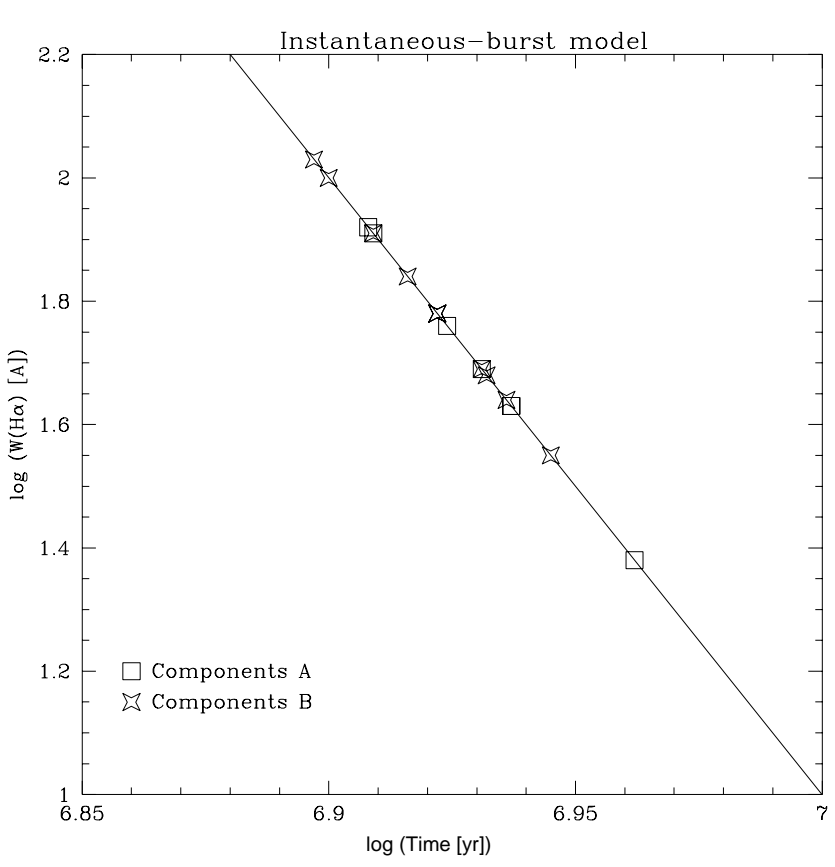

FIG. $7 b$

FIG. 7. $-(a) \mathrm{EW}(\mathrm{H} \alpha)$ vs. time for a continuous star formation model (straight line) with $Z=Z_{\odot}, \alpha=2.35$, and $M_{\mathrm{up}}=30 M_{\odot}$. Squares, A components of the physical pairs; stars, the B components. (b) Same as (a), but for an instantaneous burst model.

We also confirm the L\&K result that the morphological types of the interacting galaxies have a direct effect on the observed spectral characteristics independent of the interaction class. For instance, interactions between elliptical galaxies (group 1) tend not to affect the spectral signatures of the galaxies, i.e., they do not induce enhanced emission.

Another interesting point to consider is that, for our whole sample, we do not find any signatures of Seyfert nuclei in the spectra. One possible reason, as we pointed out in $\S 6.3$, is that strong starforming regions around the nucleus are diluting the Seyfert or LINER spectra. However, this argument cannot explain the lack of Seyfert 1 nuclei. These facts are currently being analyzed.
We are in debt with the staff of CASLEO for their excellent support. The authors gratefully acknowledge support from Fundación Antorchas. The authors also acknowledge use of the CCD and data acquisition system supported under US. National Science Foundation grant AST 90-15827 to R. M. Rich. This paper was greatly improved by the referee's comments and suggestions. Many thanks to Dr. Diego García Lambas for the English revision. This research has made use of the superb NASA/IPAC Extragalactic Database (NED) which is operated by the Jet Propulsion Laboratory, Caltech, under contract with the National Aeronautics and Space Administration.
Armus, L., Heckman, T., \& Miley, G. 1987, ApJ, 312, 566

Arp, H., \& Madore, B. F. 1987, A Catalogue of Southern Peculiar Galaxies and Associations (Cambridge: Cambridge Univ. Press) (AMC)

Bothun, G. D., Halpern, J. P., Lonsdale, C. J., Impey, C., \& Schmitz, M. 1989, ApJS, 70, 271

Bushouse, H. A. 1986, AJ, 91, 225

1987, ApJ, 320, 49

Copetti, M. V. F., Pastoriza, M. G., \& Dottori, H. A. 1986, A\&A, 156, 111

de Vaucouleurs, G., de Vaucouleurs, A., Corwin, H. G., Jr., Buta, R. J.

Paturel, G., \& Fouqué, P. 1991, Third Reference Catalogue of Bright Galaxies, (New York: Springer) (RC3)

Dahari, O. 1985, ApJS, 57, 643

Donzelli, C. J., \& Pastoriza, M. G. 1997, in preparation

Fried, J. W., \& Lutz, D. 1988, A\&A, 197, 52

Friedman, S. D., Cohen, R. D., Jones, B., Smith, H. E., \& Stein, W. A. 1987, AJ, 94, 1480

Garcia Vargas, M. L., \& Diaz, A. I. 1994, ApJS, 91, 553

Hernquist, C., \& Mihos, J. C. 1995, ApJ, 448, 41 (H\&M)

Hummel, E. 1981, A\&A, 96, 111

Jones, B., \& Stein, W. A. 1989, AJ, 98, 1557

Joseph, R. D., \& Wright, G. S. 1985, MNRAS, 214, 87

Karachentsev, I. D., \& Karachentseva, V. E. 1974, AZh, 51, 724

Keel, W. C., Kennicutt, R. C., Hummel, E., \& Van der Hulst, J. M. 1985, AJ, 90, 708

Kennicutt, R. C., \& Keel, W. C. 1984, ApJ, 279, L5

Kennicutt, R. C., Keel, W. C., Van der Hulst, J. M., Hummel, E., \& Roettiger, K. A. 1987, AJ, 93, 1011

Kennicutt, R. C., \& Kent, S. M. 1983, AJ, 88, 1094

\section{REFERENCES}

Larson, R. B., \& Tinsley, B. M. 1978, ApJ, 219, 46

Lauberts, A., \& Valentijn, E. A. 1989, The Surface Photometry Catalogue of the ESO-Uppsala Galaxies (Garching: ESO)

Leitherer, C., \& Heckman, T. M. 1995, ApJS, 96, 9 (L\&H)

Lequeux, J., Maucherat-Joubert, M., Deharveng, J. M., \& Kunth, D. 1981, A\&A, 103, 305

Liu, C. T., \& Kennicutt, R. C. 1995, ApJ, 450, 547 (L\&K)

MacKenty, J. 1989, ApJ, 343, 125

Maia, M., Pastoriza, M. G., Bica, E., \& Dottori, H. A. 1994, ApJ, 93, 425

Pastoriza, M. G., Bica, E., Maia, M., Bonatto, Ch., \& Dottori, H. A. 1994, ApJ, 432, 128

Pastoriza, M. G., \& Donzelli, C. J. 1997, in preparation

Phillips, M. M., Edmunds, M. G., Pagel, B. E. J., \& Turtle, A. J. 1983, MNRAS, 203, 759

Sekiguchi, K., \& Wolstencroft, R. D. 1992, MNRAS, 255, 581

. 1993, MNRAS, 263, 359

Sérsic, J. L., \& Pastoriza, M. G. 1965, PASP, 77, 287

Shombert, J. M., Wallin, J. F., \& Struck-Marcell, C. 1990, AJ, 99, 497

Soifer, B. T., et al. 1984, ApJ, 278, L71

Stocke, J. T. 1978, AJ, 83, 348

Stone, R. P. S., \& Baldwin, J. A. 1983, MNRAS, 204, 347

Storchi-Bergmann, T., Baldwin, J. A., \& Wilson, AS. 1993, ApJ, 410, L11

Storchi-Bergmann, T., Eracleous, M., Livio, M., Wilson, A. S., Filippenko,

A. V., \& Halpern, J. P. 1995, ApJ, 443, 617

Tonry, J. L., \& Davies, M. 1979, AJ, 84, 1511

Toomre, A., \& Toomre, J. 1972, ApJ, 178, 623

Veilleux, S., Kim, D. C., \& Kron, R. G. 1995, ApJ, 435, L105

Wasilewski, A. J. 1983, ApJ, 272, 68 